\title{
1 Space-time logic of liver gene expression at sublobular scale
}

3 Colas Droin ${ }^{1+}$, Jakob El Kholtei $^{2+}$, Keren Bahar Halpern ${ }^{2+}$, Clémence Hurni ${ }^{1}$, Milena

4 Rozenberg $^{2}$, Sapir Muvkadi $^{2}$, Shalev Itzkovitz ${ }^{2 *}$, Felix Naef $^{1^{*}}$

$6{ }^{1}$ Institute of Bioengineering, School of Life Sciences, Ecole Polytechnique Fédérale de

7 Lausanne, $\mathrm{CH}-1015$ Lausanne, Switzerland

$8{ }^{2}$ Department of Molecular Cell Biology, Weizmann Institute of Science, Rehovot 7610001,

9 Israel

$10{ }^{+}$These authors contributed equally to this work.

$11 *$ Corresponding authors:

12 Shalev Itzkovitz, Department of Molecular Cell Biology, Weizmann Institute of Science,

13 Rehovot 7610001, Israel

14 Email: shalev.itzkovitz@weizmann.ac.il; +972 89343104 :

16 Felix Naef, Institute of Bioengineering, School of Life Sciences, Ecole Polytechnique

17 Fédérale de Lausanne, CH-1015 Lausanne, Switzerland

18 Email: felix.naef@epfl.ch; phone: +41 216931621

\section{Abstract}

21 The mammalian liver performs key physiological functions for maintaining energy and 22 metabolic homeostasis. Liver tissue is both spatially structured and temporally

23 orchestrated. Hepatocytes operate in repeating anatomical units termed lobules and 24 different lobule zones perform distinct functions. The liver is also subject to extensive 25 temporal regulation, orchestrated by the interplay of the circadian clock, systemic signals 26 and feeding rhythms. Liver zonation was previously analyzed as a static phenomenon and 27 liver chronobiology at the tissue level. Here, we use single-cell RNA-seq to investigate the 28 interplay between gene regulation in space and time. Categorizing mRNA expression 29 profiles using mixed-effect models and smFISH validations, we find that many genes in 30 the liver are both zonated and rhythmic, most of them showing multiplicative space-time 
31 effects. Such dually regulated genes cover key hepatic functions such as lipid, carbohydrate

32 and amino acid metabolism, but also genes not previously associated with liver zonation

33 such as chaperones. Our data also suggest that rhythmic and localized expression of Wnt

34 targets could be explained by rhythmically expressed Wnt ligands from non-parenchymal

35 cells near the central vein. Core circadian clock genes are expressed in a non-zonated

36 manner, indicating that the liver clock is robust to zonation. Together, our comprehensive

37 scRNA-seq analysis revealed how liver function is compartmentalized spatio-temporally

38 at the sub-lobular scale.

\section{Introduction}

40 The liver is a vital organ maintaining body physiology and energy homeostasis. The liver

41 carries out a broad range of functions related to carbohydrate and lipid metabolism,

42 detoxification, bile acid biosynthesis and transport, cholesterol processing, xenobiotics

43 biotransformation, and carrier proteins secretion. Notably, the liver performs catabolic and

44 anabolic processing of lipids and amino acids and produces the majority of plasma

45 proteins $^{1}$. Liver tissue is highly structured on the cellular scale, being heterogeneous in

46 both cell-type composition and microenvironment ${ }^{2}$. In fact, liver tissue is made up of

47 millions of repeating anatomical and functional subunits, called lobules, which in mice

48 contain hepatocytes arranged in about 15 concentric layers with a diameter of about

$490.5 \mathrm{~mm}^{3,4}$. On the portal side of the lobule, blood from the portal vein and the hepatic

50 arteriole enters small capillaries called sinusoids and flows to the central vein. This is

51 accompanied with gradients in oxygen concentration, nutrients and signaling along the

52 portal-central axis, with the latter notably involving the Wnt pathway ${ }^{5,6}$. Due to this

53 polarization, hepatocytes in different layers perform separate functions. This is

54 accompanied with gradients of gene expression along the portal-central axis, with some

55 genes expressed more strongly near the central vein, and vice versa for portally expressed

56 genes. This phenomenon is termed liver zonation ${ }^{1,7}$.

57 Recently, Halpern et al. combined single-cell RNA-sequencing (scRNA-seq) of

58 dissociated hepatocytes and single-molecule RNA fluorescence in situ hybridization

59 (smFISH) to reconstruct spatial mRNA expression profiles along the portal-central axis ${ }^{8}$.

60 This analysis revealed an unexpected breadth of spatial heterogeneity, with $\sim 50 \%$ of genes 
61 showing spatially non-uniform patterns. Among them, functions related to ammonia

62 clearance, carbohydrate catabolic and anabolic processes, xenobiotics detoxification, bile

63 acid and cholesterol synthesis, fatty acid metabolism, targets of the Wnt and Ras pathways,

64 and hypoxia-induced genes were strongly zonated.

65 In addition to its spatial heterogeneity, liver physiology is also highly temporally

66 dynamic. Chronobiology studies showed that temporally gated physiological and

67 metabolic programs in the liver result from the complex interplay between the endogenous

68 circadian liver oscillator, rhythmic systemic signals, and feeding/fasting cycles $^{9,10,11}$. An

69 intact circadian clock has repeatedly been demonstrated as key for healthy metabolism,

70 also in humans ${ }^{12}$. In addition, the hepatocyte clock has specifically been shown to play a

71 major role in the physiological coordination of nutritional signals and cell-cell

72 communication (including non-hepatocytic cells) controlling rhythmic metabolism ${ }^{13}$.

73 Temporal compartmentalization can prevent two opposite and incompatible processes

74 from simultaneously occurring, for example, glucose is stored as glycogen following a

75 meal and is later released into the blood circulation during fasting period to maintain

76 homeostasis in plasma glucose levels. Functional genomics studies of the circadian liver

77 were typically performed on bulk liver tissue ${ }^{14}$. In particular, several studies showed how

78 both the circadian clock and the feeding fasting cycles pervasively drive rhythms of gene

79 expression in bulk, impacting key sectors of liver physiology such as glucose homeostasis,

80 lipid and steroid metabolism ${ }^{15-18}$.

81 Here, we asked how these spatial and temporal regulatory programs interact on the

82 level of individual genes and liver functions more generally. In particular, can zonated gene

83 expression patterns be temporally modulated on a $24 \mathrm{~h}$ time scale? And conversely, can

84 rhythmic gene expression patterns observed in bulk samples exhibit sub-lobular structure?

85 More complex situations may also be envisaged, such as time-dependent zonation patterns

86 of mRNA expression (or, equivalently, zone-dependent rhythmic patterns), or sublobular

87 oscillations that would escape detection on the bulk level due to cancelations. On the

88 physiological level, it is of interest to establish how hepatic functions might be

89 compartmentalized both in space and time. To study both the spatial and temporal axes, we

90 performed scRNA-seq of hepatocytes at four different times along the $24 \mathrm{~h}$ day, extending

91 a previous approach ${ }^{8,19}$ to reconstruct spatial profiles at each time point. The resulting 
92 space-time patterns were statistically classified using a mixed-effect model describing both

93 spatial and temporal variations in mRNA levels. In total, 5000 liver genes were classified

94 based on their spatio-temporal expression profiles, and a few representative profiles were

95 further analyzed with smFISH. Overall, this approach revealed the richness of space-time

96 gene expression dynamics of the liver and provides a comprehensive view on how spatio-

97 temporal compartmentalization is utilized at the sub-lobular scale in the mammalian liver.

\section{$98 \quad$ Results}

99 Single-cell RNA-seq captures spatiotemporal gene expression patterns in mouse liver

100 To investigate spatio-temporal gene expression patterns in mouse liver, we sequenced 101 mRNA from individual liver cells obtained via perfusion from 10 ad libitum fed mice at 4

102 different times of the day $(\mathrm{ZT}=0 \mathrm{~h}, 6 \mathrm{~h}, 12 \mathrm{~h}$ and $18 \mathrm{~h}$, two to three replicates per time point).

103 The interval between ZT0 and ZT12 has the light on and corresponds to the fasting period 104 in mice, while feeding happens predominantly between ZT12 and ZT0. We here focused 105 on hepatocytes by enrichment of cells according to size and in silico filtering, yielding a 106 total of 19663 cells (Methods). To validate that the obtained scRNA-seq data captured the 107 expected variability in both spatial and temporal mRNA levels, we generated a clustering 108 analysis of all cells using a standard 2-dimensional t-SNE dimensionality reduction 109 (Methods) and colored cells either by their positions along the central-portal axis (the a 110 posteriori assigned layers, see below) (Figure 1A) or time (Figure 1B). The clustering 111 revealed that portally and centrally expressed landmark transcripts, such as the cytochrome 112 P450 oxygenases Cyp2f2 and Cyp2el involved in xenobiotics metabolism, mark cells in 113 opposite regions of the projections (Figure 1C-D). Likewise, time-of-day gene expression 114 varied along an orthogonal direction (Figure 1B), as shown for the fatty acid elongase 115 Elovl3 peaking at ZT0 (Figure 1E).

116 To obtain spatial mRNA expression profiles for each gene along the central-portal 117 axis, we here introduced eight lobule layers, to which we assigned each individual cell. For 118 this, we adapted a previous method that uses expression levels of landmark zonated genes 119 to define a central-portal coordinate ${ }^{19}$, with the modification that only landmark transcripts 120 that were sufficiently expressed and that did not vary across mice and time points were 121 used (27 central and 28 portal landmark genes, Methods). The resulting reconstructed 
122 (binned) mRNA expression profiles yielded 80 (8 layers over 10 mice) data points for each 123 transcript. Although our resolution is lower compared with the typically 12-15 hepatocyte 124 layers found in the liver ${ }^{3,4}$, these reconstructions faithfully captured reference zonated 125 genes, with both central, and portal, expression (Figure 1F). Two examples of such genes 126 are the centrally expressed glutamine synthethase $(G l u l)$, and the portally expressed urea 127 cycle gene argininosuccinate synthetase (Ass 1 ), showing mutually exclusive expression 128 along the lobule ${ }^{8}$. The reconstruction also successfully identified transcripts of the core 129 circadian clock, such as the master transcription factor Bmall (also named Arntl), whose 130 mRNA peaked between ZT18-ZT0 (Figure 1G) ${ }^{20}$. In addition to core clock genes, 131 important clock outputs such as the PAR bZip transcription factor Dbp, which is a direct 132 transcriptional target of BMAL1 regulating detoxification enzymes, peaked between ZT613312 (Figure $1 G)^{21}$. Finally, genes showing both zonated and rhythmic mRNA accumulation 134 were found (Figure 1H), for example elongation of very long chain fatty acids 3 (Elovl3) 135 is centrally expressed and peaks near ZT0, while phosphoenolpyruvate carboxykinase 1 $136(P c k 1)$ regulating gluconeogenesis during fasting is expressed portally and peaks shortly 137 before ZT12. Since most of the zonated profiles showed exponential shapes, and gene 138 expression changes typically occur on a $\log \operatorname{scal}^{22}$, we log-transformed the data for further 139 analysis (Methods, Figure $1 \mathrm{~F}$ and Supplementary Figure 1G-H-I). Together, these 140 examples indicate that the obtained gene expression profiles reliably capture spatial and 141 temporal regulation of hepatocyte gene expression. 
Figure 1. A scRNA-seq approach to space-time gene expression in mouse liver.

A

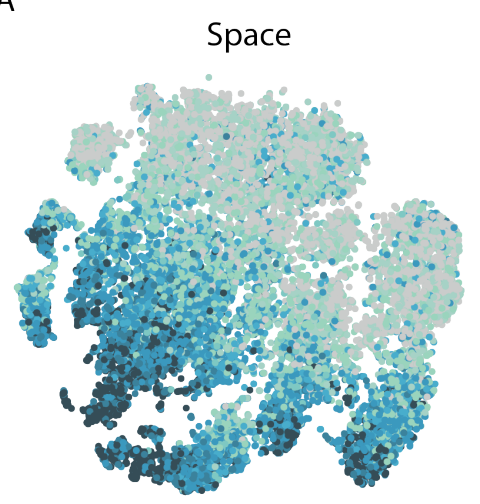

C

Cyp2f2

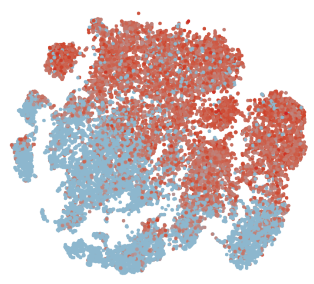

$\mathrm{F}$

Zonated
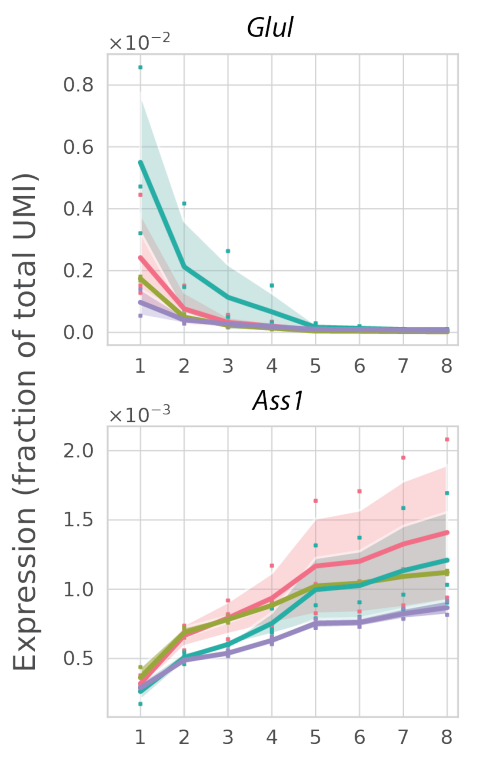

D

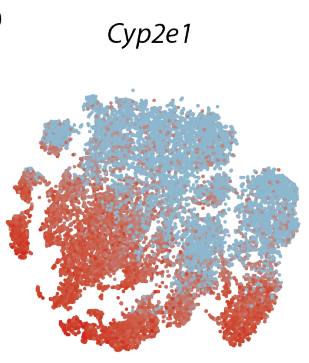

G

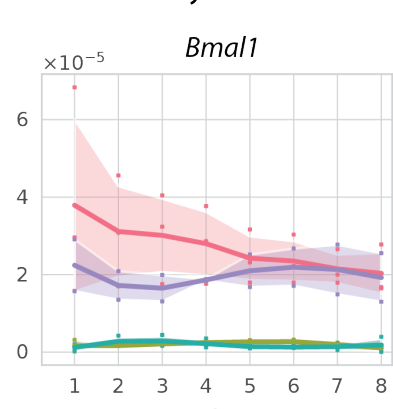

Dbp

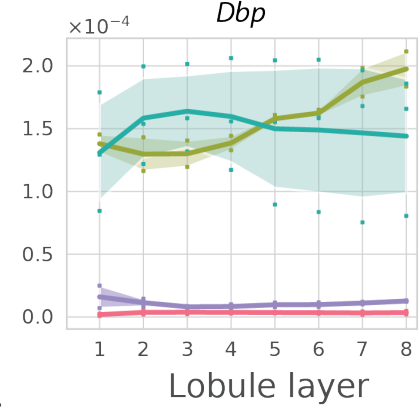

PC
B

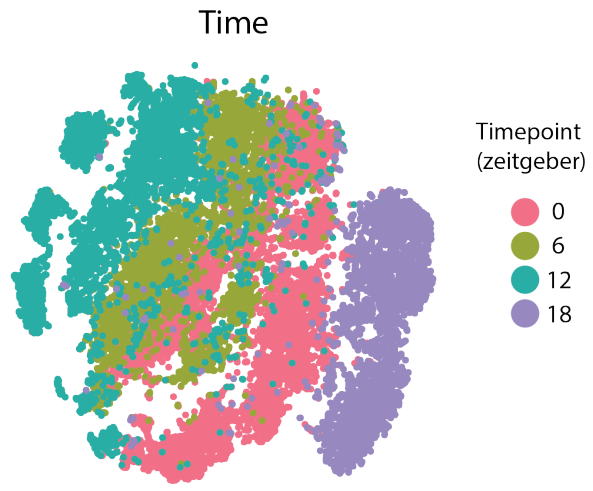

144 (A-E) Global gene expression varies in both space and time, as shown using $t$-SNE visualizations of the scRNA-seq (19663 hepatocytes from 10 mice). Each dot represents

E Elov/3

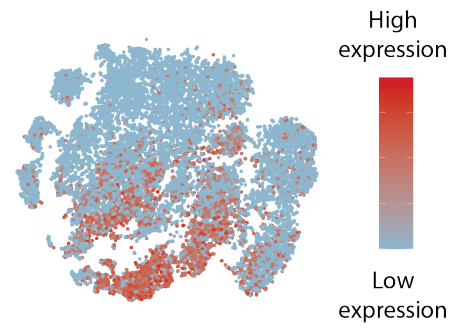

H

Zonated and rhytmic

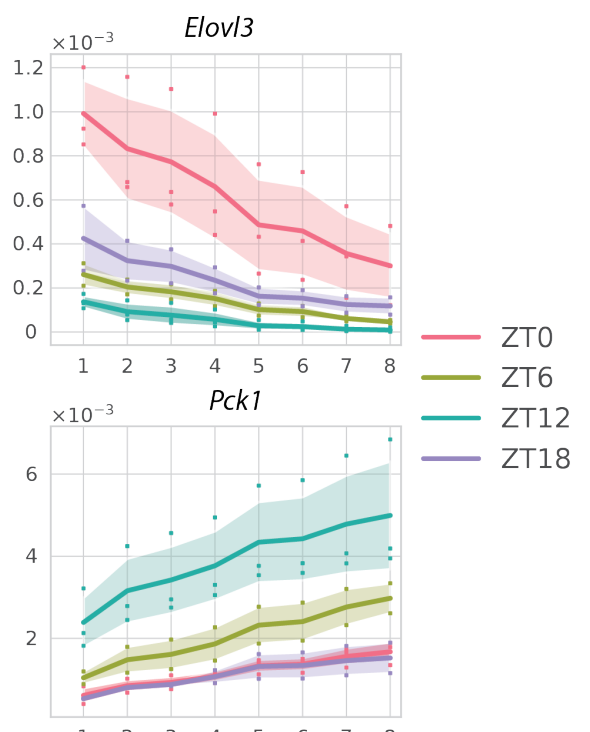
PP one cell. Individual cells are colored by the (a posteriori assigned) lobule layer (A), 
147 Zeitgeber time (B), expression levels of the zonated genes Cyp2f2 and Cyp2e1 (C-D), or 148 the temporally-regulated and centrally zonated gene Elovl3 (E).

149 (F-H) Reconstructed spatial profiles (lobule layers 1-8) of selected zonated genes (F, top: Glul pericentrally (PC) expressed, bottom: Ass 1 periportally (PP) expressed); rhythmic but non-zonated genes ( $\mathrm{G}$, top: core-clock gene Bmall peaking at ZT18-0, bottom: clockcontrolled Dbp, peaking at ZT6-12); zonated and rhythmic genes (H, Top: Elovl3, bottom: Pck1). Expression levels correspond to fraction of total UMI per cell in linear scale. Logtransformed profiles are in Supplementary Figure 1. Dots in F-H represent data points from

Space-time mRNA expression profiles categorized according to zonation and rhythmicity

159 To gain a systematic understanding of the space-time gene expression profiles, we next 160 investigated if zonated gene expression patterns could be dynamic along the day, or conversely whether temporal expression patterns might be zone-dependent. To select a reliable set of reconstructed mRNA expression profiles for subsequent analyses, we filtered

163 out lowly expressed genes as well as genes with significant biological variability across 164 replicate liver samples, although this may be at the expense of a potentially decreased 165 sensitivity (Methods). This yielded 5058 spatio-temporal gene expression profiles 166 (Supplementary Figure 2A). An exploratory analysis of variance clearly identified zonated 167 genes, rhythmic genes, and fewer genes showing variability along both axes, with known 168 zonated and rhythmic genes distributed as expected (Figure 2A).

169 To identify possible dependencies between spatial and temporal variations, we built 170 a mixed-effect linear model ${ }^{23}$ for the space-time mRNA profiles, which extends harmonic 171 regression to include a spatial covariate (Figure 2B). In this model, rhythms are

172 parameterized with cosine and sine functions, while spatial profiles are represented with 173 (up to second order) polynomials. In its most complex form, the model uses nine 174 parameters describing spatially modulated oscillations, and one intercept per mouse 175 (Methods). When some of the parameters are zero, the model reduces to simpler mRNA 176 profiles, for example purely spatial or purely temporal expression profiles (Figure 2C). We 177 then used model selection ${ }^{24}$ to identify the optimal parameterization and category for each 
178 gene (Methods). In fine, we classified each mRNA profile into one of five types of patterns

179 (Figure 2C). If only the intercept is used, the profile will be classified as flat or noisy (F/N,

180 Methods). If only time-independent zonation parameters are retained, the predicted profile

181 will be purely zonated (Z). If only layer-independent rhythmic parameters are retained, the

182 predicted profile will be purely rhythmic (R). If only layer-independent rhythmic

183 parameters and time-independent zonation parameters are retained, the profile is classified

184 as independent rhythmic-zonated $(\mathrm{Z}+\mathrm{R})$. If at least one layer-dependent rhythmic

185 parameter is selected, the profile will be termed interacting (ZxR). This classification

186 revealed that, overall, about $30 \%$ of the mRNA profiles were zonated $(\mathrm{Z}, \mathrm{Z}+\mathrm{R}$ and $\mathrm{ZxR})$

187 and about $20 \%$ were rhythmic ( $R, Z+R$ and $Z x R$ ) (Figure 2D). The peak times of these

188 rhythmic transcripts were highly consistent with bulk chronobiology data ${ }^{25}$

189 (Supplementary Figure 2B). The entire analysis can be browsed as a web-app resource

190 along with the corresponding data (https://czviz.epfl.ch).

191 Interestingly, we found that $7 \%$ of the analyzed genes in the liver were both zonated and

192 rhythmic. Such dually regulated transcripts represent $25 \%$ of all zonated transcripts, and

$19336 \%$ of all rhythmic transcripts, respectively. For example, the previously shown Elovl3

194 transcript, involved in fatty acid elongation, and Pck1, a rate limiting enzyme in

195 gluconeogenesis, are prototypical Z+R genes (Figure 1H, Supplementary Figure 1H).

196 Gluconeogenesis is an energetically-demanding task ${ }^{3}$. As mice are in a metabolically fasted

197 state requiring glucose production towards the end of the light phase ( ZT10) and oxygen

198 needed for ATP production is most abundant portally ${ }^{26}$, this process is indeed both spatially

199 and temporally regulated. The dual regulation of zonated-rhythmic genes may therefore

200 ensure optimal liver function under switching metabolic conditions.

201 Dually regulated genes were mostly Z+R, with only a minority of ZxR patterns.

202 The average expression across categories showed that rhythmic genes are significantly less

203 expressed on average than genes in zonated categories, likely reflecting shorter half-lives

204 (Figure 2E and Supplementary Figure 2C). Surprisingly, we find few highly expressed flat

205 genes. Together, we found that mRNA expression of many zonated genes in hepatocytes

206 is not static, and is in fact compartmentalized both in space and time. 
Figure 2. Space-time mRNA expression profiles categorized with mixed-effect models.

A

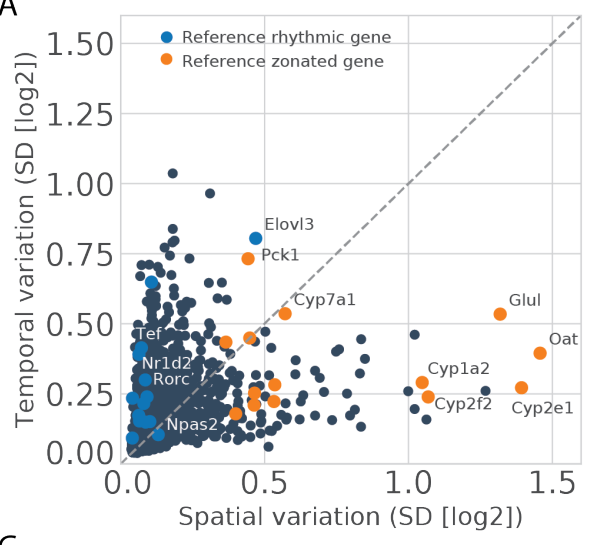

B

$$
\begin{aligned}
& y_{x, t, i}= \mu_{i}: \text { animal-specific random-effect } \\
&\left.\begin{array}{l}
+\mu(x): \text { static zonation } \\
+a(x) \cos (\omega t) \\
+b(x) \sin (\omega t)
\end{array}\right\}: \text { zone-dependent rhythmicity } \\
&+\epsilon_{x, t, i}: \text { noise }
\end{aligned}
$$

With: $\quad \mu, a, b$ : polynomials $\omega=\frac{2 \pi}{24}:$ angular frequency $\left(\mathrm{h}^{-1}\right)$

C

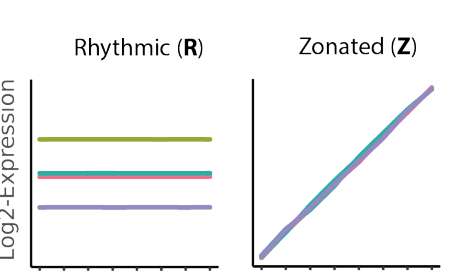

Zonated and Rhythmic

Zonated and Rhythmic

Example of $\mathbf{Z}+\mathbf{R}$ gene

(Elovl3)

Example of ZxR gene Additive (Z+R)
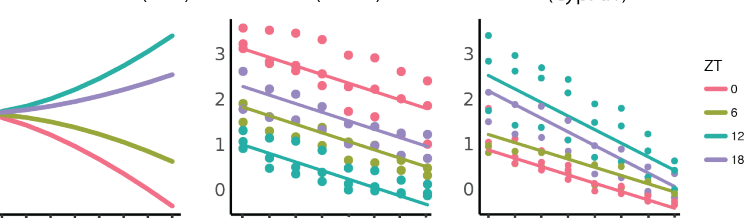

$\mathrm{PC} \longrightarrow$ Lobule layer $\mathrm{PP}$
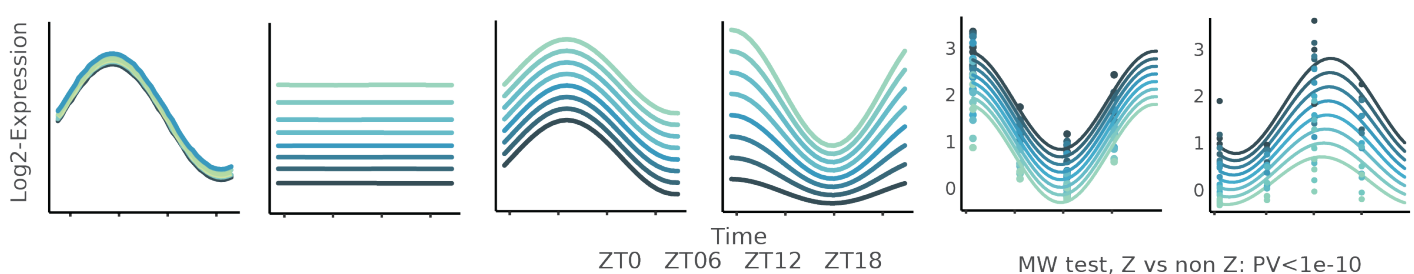

MW test, $Z$ vs non Z: PV $<1$ e- 10 $M W$ test, $R$ vs non $R: P V<1 e-10$

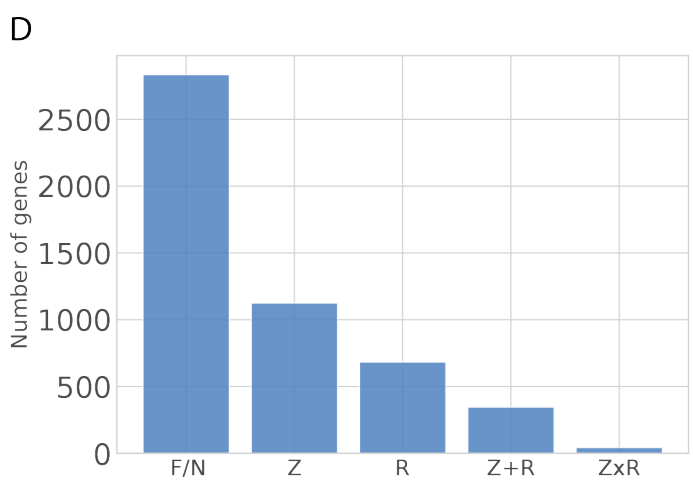

E KW test: $P V<1 e-10$

(A) Spatial and temporal variations for mRNA transcript profiles, calculated as standard deviations (SD) of $\log 2$ expression along spatial or temporal dimensions. Colored dots correspond to reference zonated genes (orange) and reference rhythmic genes (blue) (Methods). 
214 (B) Extended harmonic regression model for spatio-temporal expression profiles 215 describing a static but zonated layer-dependent mean $\mu(x)$, as well as layer-dependent 216 harmonic coefficients $(a(x)$ and $b(x))$. All layer-dependent coefficients are modeled as 217 second order polynomials; $i$ denotes the biological replicates. Temporal dependency is 218 modelled with $24 \mathrm{~h}$-periodic harmonic functions. $\mu_{i}$ are random effects needed due to the 219 data structure hierarchy (Methods).

220 (C) Schema illustrating the different categories of profiles. Depending on which coefficients are non-zero (Methods), genes are assigned to: $\mathrm{F} / \mathrm{N}$ (flat or noisy, not represented), Z (zonated), R (rhythmic), Z+R (additive zonation and rhythmicity), ZxR (interacting zonation and rhythmicity). Graphs emphasize either zonation (top), with the xaxis representing layers, or rhythmicity (bottom), with the $\mathrm{x}$ axis representing time (ZT). Right side of the panel: two examples of fits (Elovl3 and Cyp7a1, respectively Z+R and ZxR).

(D) Number of transcripts in each category.

(E) Boxplot of the mean expression per category shows that zonated genes $(\mathrm{Z}, \mathrm{Z}+\mathrm{R}$ and $\mathrm{ZxR})$ are more expressed than rhythmic $(\mathrm{R})$ or flat/noisy $(\mathrm{F} / \mathrm{N})$. ZxR genes are the most expressed according to median expression (orange line). Box limits are lower and upper quartile, whiskers extend up to the first datum greater/lower than the upper/lower quartile plus 1.5 times the interquartile range. Remaining points are outliers. KW test stands for

235 Properties of dually zonated and rhythmic mRNA profiles

236 The majority of dually regulated genes are $\mathrm{Z}+\mathrm{R}$, which denotes additive (in $\log$ ) space237 time effects, or dynamic patterns where slopes or shapes of spatial patterns do not change 238 with time (Figure 2C). On the other hand, interacting patterns (ZxR) are rare. Comparing 239 the proportions of central, mid-lobular (peaking in the middle of the portal-central axis) 240 and portal genes among the purely zonated genes (Z), and independently zonated and 241 rhythmic genes $(Z+R)$, did not reveal significant differences (Figure 3A), suggesting that 242 rhythmicity is uncoupled with the direction of zonation. Similarly, comparing the phase 243 distribution among the purely rhythmic genes $(\mathrm{R})$ and the $\mathrm{Z}+\mathrm{R}$ genes did not show a 244 significant difference (Figure 3B), indicating that zonation does not bias peak expression 
245 time. Moreover, oscillatory amplitudes were uncorrelated with the zonation slopes in $\mathrm{Z}+\mathrm{R}$ 246 genes (Figure 3C). Finally, for ZxR genes with potentially more complex space-time 247 patterns, we investigated the spreads in amplitudes and peak times across the layers (Figure 248 3D). For wave-like pattern (phase modulated profiles), the phase difference across the 249 lobule was up to $3 \mathrm{~h}$, which corresponds to a difference in time between neighboring 250 hepatocytes on the order of 10 minutes for lobules of about 15 cell layers. On the other 251 hand, amplitude modulated patterns showed up to 2-fold difference in oscillatory amplitude 252 across the lobule.

253 To assess the potential physiological role of dually zonated and rhythmic 254 transcripts, we asked if protein levels of the identified $\mathrm{Z}+\mathrm{R}$ and $\mathrm{ZxR}$ genes accumulated 255 rhythmically in a previous proteomics experiment ${ }^{27}$. In general, proteins rhythms are fewer, 256 damped, and time-delayed compared to mRNA rhythms due to protein half-lives ${ }^{14,27,28}$ 257 (Discussion). However, while R transcripts were twice more frequent than $\mathrm{Z}+\mathrm{R}$ transcripts, 258 the proportions were inverted for rhythmic proteins. Indeed, we found that among 65 259 rhythmic proteins (with q-value $<0.2$ in ref. $^{27}$ ), 18 corresponded to $\mathrm{Z}+\mathrm{R}$ and 10 to $\mathrm{R}$ 260 transcripts. Moreover, the identified $\mathrm{Z}+\mathrm{R}$ and $\mathrm{ZxR}$ genes with rhythmic protein 261 accumulation cover key hepatic and zonated functions (Supplementary Figure 3, for a 262 functional interpretation, see below) and include rate limiting enzymes. For example, for $263 \mathrm{Z}+\mathrm{R}$ transcripts (Supplementary Figure 3A), PCK1 (rate limiting for gluconeogenesis), 264 LPIN2 (Lipin2, catalyzes the conversion of phosphatidic acid to diacylglycerol during 265 triglyceride, phosphatidylcholine and phosphatidylethanolamine biosynthesis), POR 266 (cytochrome P450 oxidoreductase, required to activate P450 enzymes), DNAJA1 (HSP40 267 co-chaperone), ALAS1 (rate-limiting for heme biosynthesis), GNE (rate-limiting in the 268 sialic acid biosynthetic pathway), THRSP (biosynthesis of triglycerides from medium269 length fatty acid chains), show robust rhythms at the protein level. Similarly, for ZxR 270 proteins, CYP7A1 (rate limiting enzyme in bile acid synthesis), CYP2A5 (coumarin 7271 hydroxylase), SLC1A2 (high-affinity glutamate transporter), and multidrug resistance 272 protein ABCC2 show rhythms on the protein level (Supplementary Figure 3B). Moreover, 273 the protein rhythms accompanying those $\mathrm{Z}+\mathrm{R}$ and $\mathrm{ZxR}$ transcripts peak with an expected 274 delay of maximally about 6 hours $^{28}$ compared to the mRNA peak times (Supplementary 275 Figure 3C). 
Figure 3. Properties of dually zonated and rhythmic mRNA profiles.

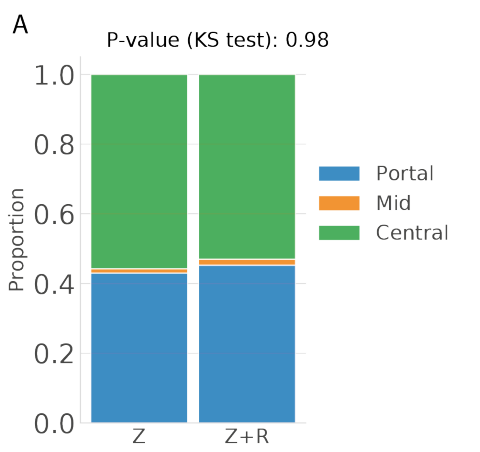

C
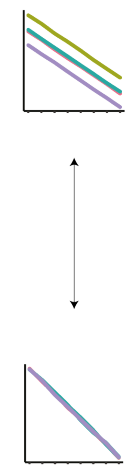

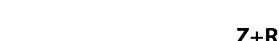

$\mathbf{Z}+\mathbf{R}$

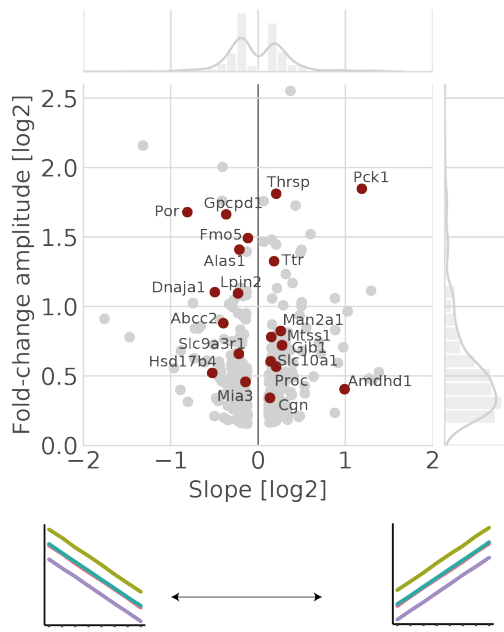

B
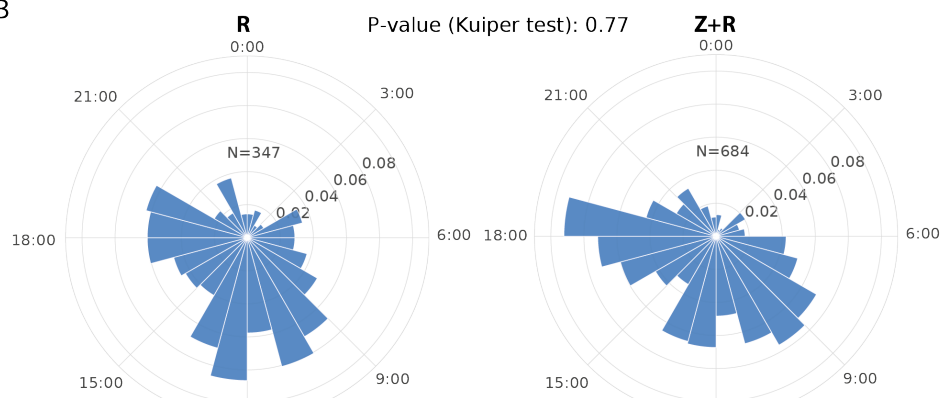

$12: 00$

D

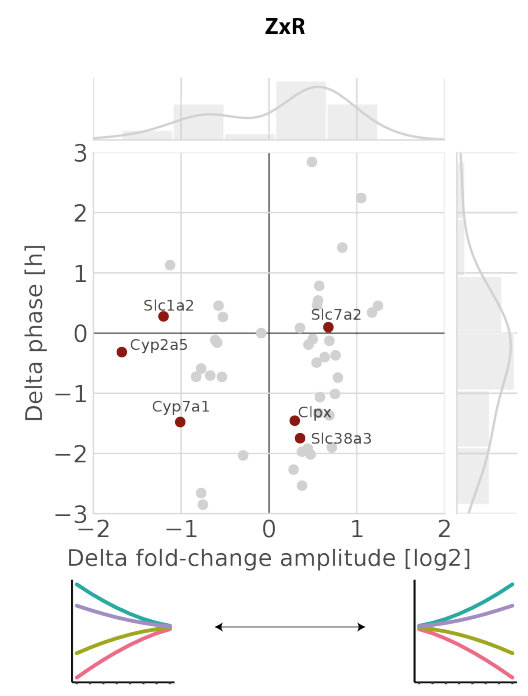

(A) Proportions of pericentral (green) and periportal (blue) transcripts are similar in Z and $\mathrm{Z}+\mathrm{R}$. Mid-lobular genes (orange) are rare $(<2 \%)$.

(B) Peak time distributions of rhythmic transcripts are similar in $\mathrm{R}$ and $\mathrm{Z}+\mathrm{R}$ categories.

(C-D) Effect sizes of zonation (slope) vs. rhythmicity (fold-change amplitude, $\log 2$ peakto-trough) in $\mathrm{Z}+\mathrm{R}$ genes $(\mathrm{C})$. Magnitude of time shifts (delta phase, in hours) $v s$. foldchange amplitude gradient (delta amplitude, in $\log 2$ ) along the central-portal axis in ZxR genes (D). Genes for which the protein is also rhythmic $(p<0.05)$ in bulk data are indicated in dark red with the corresponding label (represented in Supplementary Figure 3). 


\section{7 smFISH analysis of space-time mRNA counts}

288 To substantiate the RNA-seq profiles, we performed RNA single molecule fluorescence in

289 situ hybridization (smFISH) experiments on a set of selected candidate genes with diverse

290 spatio-temporal patterns. smFISH provides a sensitive and independent, albeit low-

291 throughput, measurement of mRNA expression. Purely zonated genes (Z) were already

292 well studied with $\mathrm{smFISH}^{8}$. To analyze the core-clock, we measured two genes peaking at

293 different times, Bmall and Perl, which were classified as R in the RNA-seq analysis.

294 Bmall ( ZT0) and Perl ( ZT12) phases were nearly identical in both experiments, and the

295 rhythms did not depend on the lobular position consistent with R genes (Figure 4A). We

296 analyzed three genes classified as Z+R: Pckl was indeed both portally biased and rhythmic

297 in RNA-seq and smFISH (Figure 4B); Elovl3 is both centrally biased and rhythmic in

298 RNA-seq and smFISH, even though the amplitude of the oscillations was damped on the

299 portal side in the FISH experiment (Supplementary Figure 4A); and for Arg1 (Arginase 1)

300 the portal RNA-seq and smFISH profiles matched well (Supplementary Figure 4B).

301 Finally, Acly showed a pattern in smFISH data which validates its classification as ZxR,

302 with a lower amplitude on the portal side, where the transcript is more highly expressed

303 (Figure 4C). Thus, overall, the reconstructed scRNA-seq and smFISH profiles were

304 consistent, with minor discrepancies. 
bioRxiv preprint doi: https://doi.org/10.1101/2020.03.05.976571; this version posted October 9, 2020. The copyright holder for this preprint (which was not certified by peer review) is the author/funder, who has granted bioRxiv a license to display the preprint in perpetuity. It is made available under aCC-BY-NC-ND 4.0 International license.

\section{$306 \quad$ Figure 4. smFISH analysis of rhythmic and zonated transcripts.}

A

ZTO

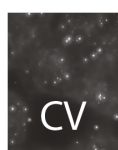

ZT6

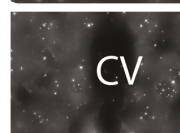

$\therefore$

ZT12

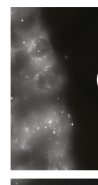

ZT18

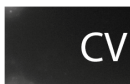

\section{CV}

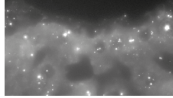

B

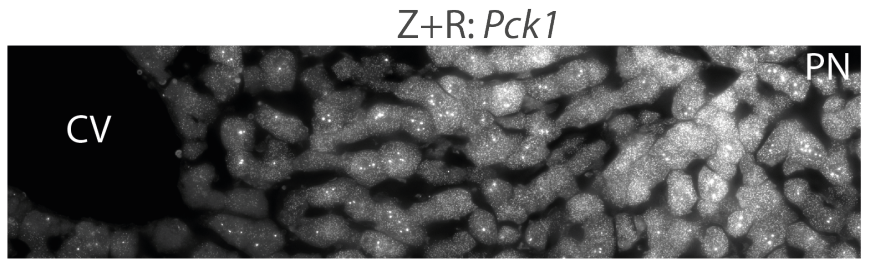

ZTO 5.

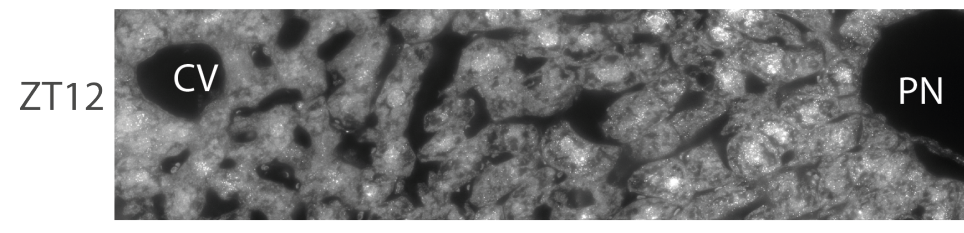

C

$$
\text { ZxR: Acly }
$$

ZTO

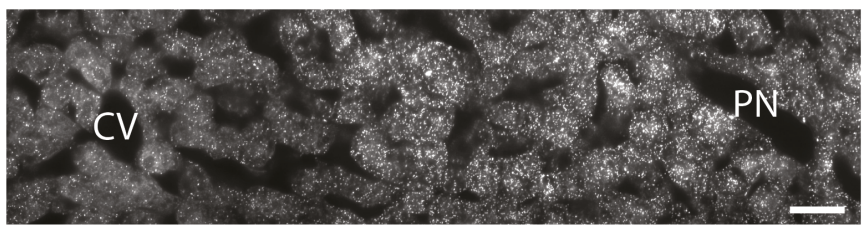

ZT12

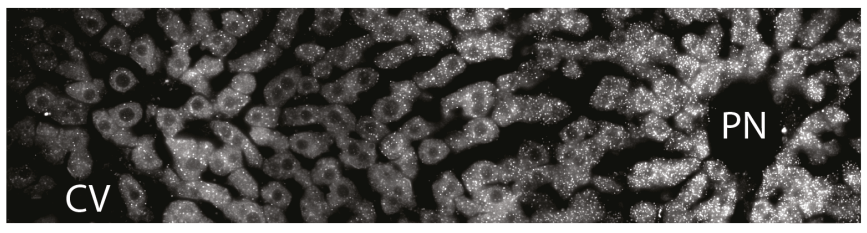

Pmal1 and Perl (smFISH)

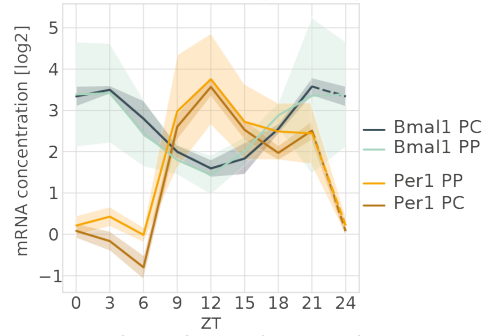

PtS.
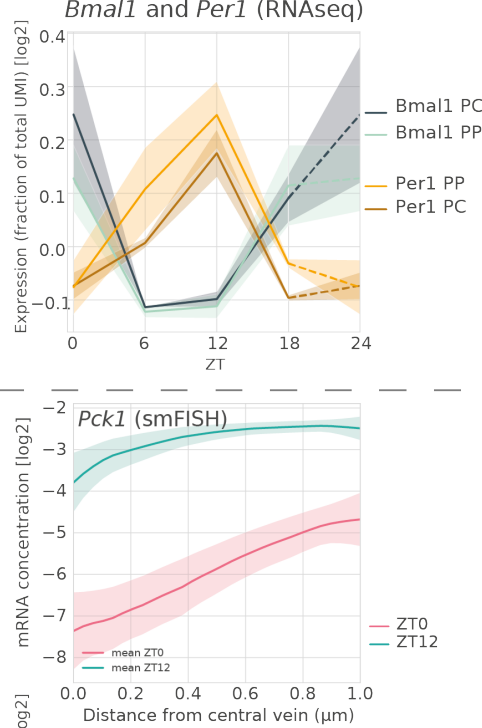

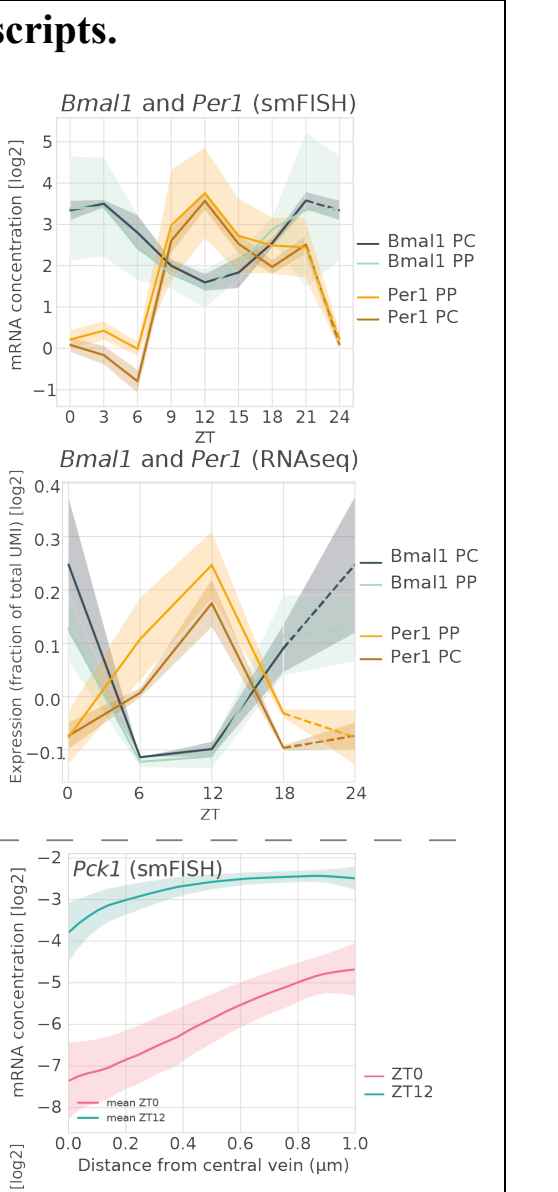

$\sum_{\overline{\bar{\Xi}}} 6.0$ PCK1 (RNAseq)

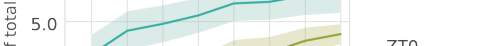

- ZTO

登 4.0

蛋 3.5

3.0

2.5

$\begin{array}{llllllll}1 & 2 & 3 & 4 & 5 & 6 & 7 & 8\end{array}$

$\widehat{N}-3.0$ Acly (smFISH)

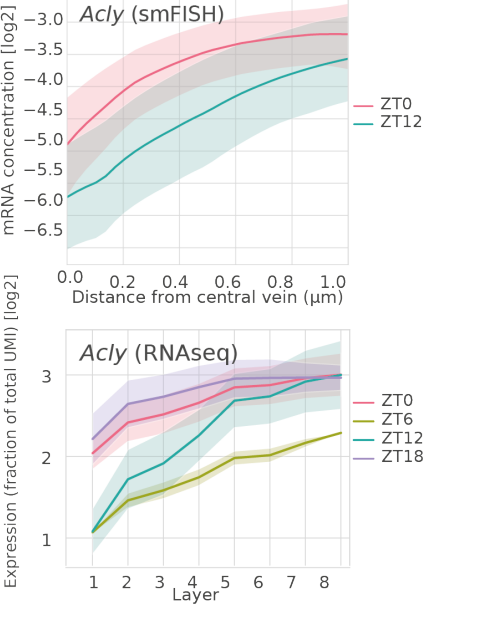


308 (A) smFISH (RNAscope, Methods) of the core clock genes Bmall and Perl (both assigned 309 to R) in liver slices sampled every 3 hours. Left: representative images at ZT0, ZT06, ZT12 310 and ZT18 for Bmall. Central vein (CV) and a portal node (PN) are marked. Scale bar is $31150 \mu \mathrm{m}$. Endothelial cells lining the PC and cholangiocytes surrounding the PP were 312 excluded from the quantification. mRNA transcripts and nuclei were detected in PN and 313 PC zones (Methods). Right: temporal profiles of BmallI and Perl from smFISH (top, 314 quantification of the number of mRNA transcripts at 8 time points ZT0 to ZT21, every 3 315 hours, shaded area indicate SD across images), in PN and PC regions, and scRNA-seq 316 (bottom, shaded areas is SD across mice).

317 (B-C) smFISH (Stellaris, Methods) for Pckl (Z+R) and Acly (ZxR). smFISH 318 quantifications were made for ZT0 and ZT12 (Methods). Left: representative images at 319 ZT0, ZT12 for Pckl (B) or Acly (C). Central vein (CV) and a portal node (PN) are marked. 320 Scale bar - $20 \mu \mathrm{m}$. Right: quantified profiles for each gene in the two time points from smFISH (top, shaded area indicate SD across images), and scRNA-seq data at the four time points (bottom, shaded areas is SD across mice).

325 We next used our classification to explore the spatio-temporal dynamics of hepatic 326 functions and signaling pathways in the liver. Given the prevalence of zonated gene 327 expression profiles, we first analyzed if the circadian clock is sensitive to zonation. We 328 found that profiles of reference core-clock genes (Supplementary Figure 5) were assigned 329 to the rhythmic only category (R), except for Cryl and Clock that were assigned to Z+R, 330 but with high probabilities also for R (Supplementary Table 2). This suggests that the 331 circadian clock is largely non-zonated, as also seen in the smFISH (Figure 4A), and 332 therefore robust to the heterogenous hepatic microenvironment.

333 We then systematically explored enrichment of biological functions in the zonated 334 category by querying the KEGG pathways database (Supplementary Table 3, Figure 5, 335 Methods). In addition to recapitulating well documented zonated liver functions ${ }^{8,29}$, which 336 we do not discuss here, this analysis highlighted $\mathrm{Z}$ and $\mathrm{Z}+\mathrm{R}$ functions which, to our 337 knowledge, had not been linked with liver zonation. For instance, we found that cytosolic 338 chaperones accumulate centrally, while the endoplasmic reticulum (ER) chaperones linked 
339 with protein secretion accumulate portally (Figure 5B,D, Supplementary Figure 6). Both 340 groups of chaperones peak during the activity/feeding phase, probably due to body 341 temperature rhythms peaking during the active phase ${ }^{30,31}$, and likely reflect increased needs

342 of protein folding during times of high protein synthesis. Also, we found that mRNAs of 343 ribosomal protein genes accumulate centrally $(Z)$, as do proteasome components (Figure $3445 \mathrm{~A})$, the latter also containing rhythmic members $(\mathrm{Z}+\mathrm{R})$. In the liver, ribosomal proteins 345 are rate-limiting for the synthesis of ribosomes, which themselves are rate-limiting for the 346 synthesis of proteins ${ }^{32}$. Therefore, the overall protein synthesis rate is likely higher in these 347 hepatocytes. Conversely, transcripts encoding components of the proteasome are involved 348 in protein degradation. Together, these observations suggest that protein turnover is higher 349 in centrally located hepatocytes, which are exposed to an environment with high 350 concentrations of xenobiotics and hypoxic stress ${ }^{33}$.

351 In addition, while many mitophagy genes are expressed centrally (Z), some of those 352 also show robust temporal rhythms peaking during the fasting period $(Z+R)$, in particular 353 two gamma-aminobutyric acid receptor-associated proteins (Gabarap and Gabarapll) 354 with an important function in autophagosome mediated autophagy ${ }^{34}$. Consistent with this 355 temporal regulation, we had previously reported that the nuclear abundance of the fasting356 dependent regulators of autophagy TFEB and ZKSCAN3 peaked near ZT6 ${ }^{35}$. Moreover, 357 the centrally and synchronously accumulating Ubiquitin B mRNA (Ubb, Z+R) may 358 contribute to triggering mitophagy ${ }^{36}$. Thus, centrally biased mitophagy may both 359 participate in removal of damaged mitochondria in the stressed central environment.

360 Similarly, genes involved in bile acid synthesis and bile secretion are known to 361 show zonated expression patterns ${ }^{8}$ (Figure 5C). Here, we found that while the rate-limiting 362 enzyme in the bile acid biosynthetic pathway Cyp7al (ZxR, Figure 2C) is known to be 363 clock-controlled, with its $\mathrm{mRNA}^{37,38}$ and protein $^{39}$ (Supplementary Figure 7D) expressed 364 maximally early during the feeding period, the ABC transporters Sterolin-1 and 2 (ABCG5/ 365 ABCG8, both identified as Z+R) which excrete most of the biliary cholesterol ${ }^{40}$ peak 366 towards the end of the fasting period near ZT9.

367 Many detoxification enzymes of the cytochromes P450 (CYPs) superfamily are 368 known to be centrally zonated in the liver $^{8}$ and several of those are found in the $\mathrm{Z}+\mathrm{R}$ 369 category. In particular, the Flavin-containing monooxygenases Fmo1, 2, and 5, which are 
370 NADPH-dependent monooxygenases involved in drug and xenobiotics detoxification, 371 exhibit Z+R mRNA patterns with peaks near ZT16 (Figure 5C). Also, the FMO5 protein

372 accumulates rhythmically (Figure 3C, Supplementary Figure 3). Moreover, the rate

373 limiting enzyme ALAS1 producing the P450 cofactor heme was found as a centrally

374 zonated Z+R transcript with peak mRNA at ZT13, showing also a robust rhythm in protein 375 expression (Supplementary Figure 3).

376 To substantiate the above finding on heat shock genes, we examined the space-time

377 behavior of temperature-regulated genes. To this end, we considered targets (bound in

378 ChIP-seq) of the heat shock transcription factor HSF1 (Chip-Atlas ${ }^{41}$, includes our own liver

379 data $^{16}$ ). This showed that several targets, known to peak during the active phase ${ }^{30,31}$, are

380 also zonated (Supplementary Figure 6). Notably, the cytoplasmic Hsp90aal (Hsp90A

381 chaperone) and its interactor Dnajal (HSP40), respectively mitochondrial Hspd1 (HSP60),

382 chaperones are expressed centrally where protein turnover is high. On the contrary,

383 endoplasmic reticulum (ER) located chaperones: Hspb1 (Hsp90B) and interactor Pdia3, as

384 well as Hspa5 (HSP70) and ER-resident Dnajc3 and Dnajb11 (DNAJ/HSP40) are

385 expressed portally, consistent with their role in folding proteins in the secretory pathway

386 (secretion is known to occur portally ${ }^{29}$ ). On the other hand, the analysis of cold induced 387 genes (i.e. CIRBP and analogous, taken from ref. ${ }^{42}$ ) did not show zonated gene expression.

388 Finally, we note that among all KEEG pathway related to lipids, a majority show 389 central enrichment (Figure 5A, Table S3). Inspection of the genes involved shows that this 390 is due to the large number of genes related to peroxisomal $\beta$-oxidation, i.e. lipid catabolism, 391 which are incidentally also listed in biosynthesis KEGG pathways (Table S3). However, 392 fatty acid synthesis is biased portally, as supported by key portally expressed genes such 393 as Fasn, Srebf1, Acly, Acaca, Elovl2, Elovl5, and which is consistent with the fact that 394 oxygen needed for mitochondrial $\beta$-oxidation is most abundant portally ${ }^{26}$. On the other 395 hand, Elovl3, which is known to be transcriptionally controlled by the peroxisome regulator 396 PPAR $\alpha$ and atypically regulated among Elov family fatty acid elongases ${ }^{43}$ is expressed 397 centrally. 
bioRxiv preprint doi: https://doi.org/10.1101/2020.03.05.976571; this version posted October 9, 2020. The copyright holder for this preprint (which was not certified by peer review) is the author/funder, who has granted bioRxiv a license to display the preprint in perpetuity. It is made available under aCC-BY-NC-ND 4.0 International license.

A

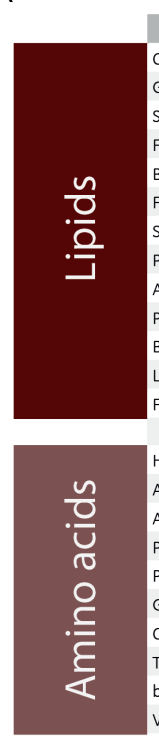

Term
Cholesterol metabolism
Glycerophospholipid metabolism
Steroid biosynthesis
Fat digestion and absorption
Biosynthesis of unsaturated fatty

Fatty acid elongation *

Steroid hormone biosynthesis

PPAR signaling pathway

Arachidonic acid metabolism

Peroxisome

Butanoate metabolism

inoleic acid metabolism

atty acid degradation

Histidine metabolism

Arginine biosynthesis

Alanine, aspartate and glutamate metabolism

Phenylalanine metabolism

Glycine, serine and threonine metabolism

Cysteine and methionine metabolism

Tryptophan metabolism

beta-Alanine metabolism

Valine, leucine and isoleucine degradation

$\begin{array}{cl}\text { Protein } & \begin{array}{l}\text { Protein processing in endoplasmic reticulum } \\ \text { Ribosome }\end{array} \\ \text { homeostasis } & \text { Proteasome }\end{array}$

Carbohydrates $\begin{aligned} & \text { Pyruvate metabolism } \\ & \text { Pentose and glucuronate interconversions }\end{aligned}$

Insulin Glucagon signaling pathway

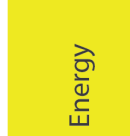

AMPK signaling pathway

Oxidative phosphorylation

Citrate cycle (TCA cycle)

Nicotinate and nicotinamide metabolism

Glyoxylate and dicarboxylate metabolism

Synthesis and degradation of ketone bodies

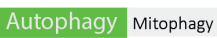

\section{Vitamin}

Ascorbate and aldarate metabolism

Retinol metabolism

Heme Porphyrin and chlorophyll metabolism

Lysosome Lysosome

Bile

Bile secretion

Primary bile acid biosynthesis

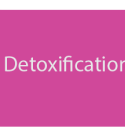

Drug metabolism

Glutathione metabolism

Metabolism of xenobiotics by cytochrome P450

Chemical carcinogenesis

Iron Ferroptosis

Complements Complement and coagulation cascades

400

401

402

403
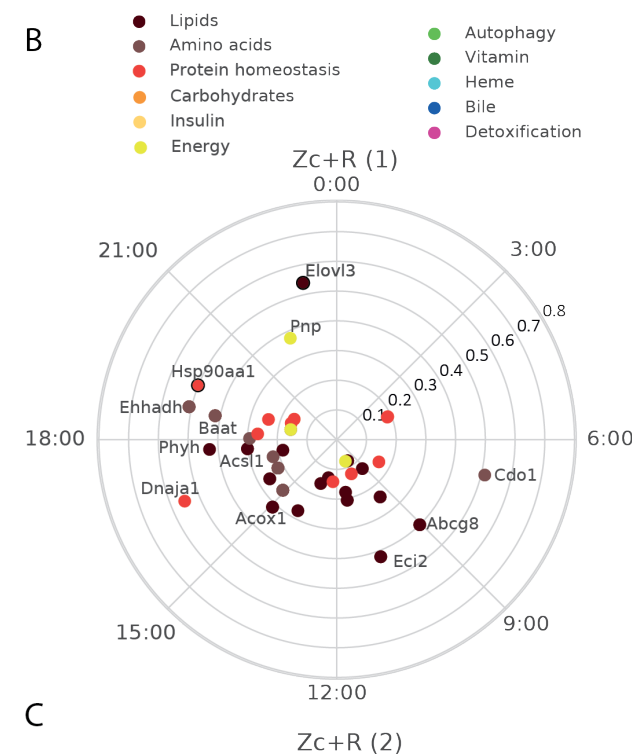

\begin{tabular}{|c|c|}
\hline $1.41 E-05$ & 35 \\
\hline $1.41 E-09$ & 100 \\
\hline $1.67 E-04$ & 100 \\
\hline
\end{tabular}

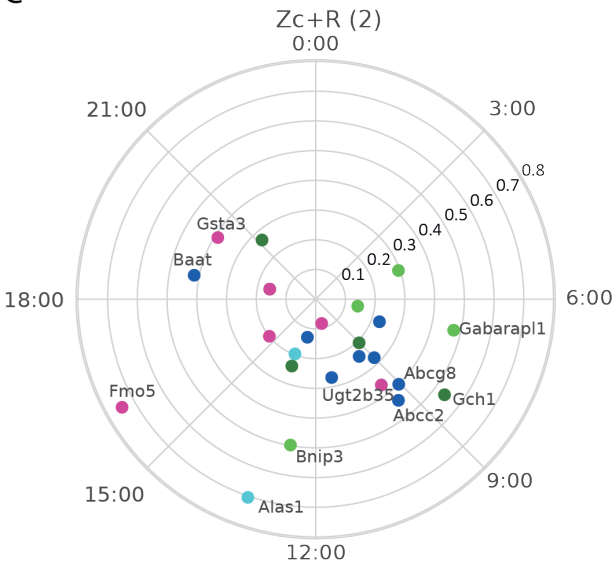

D
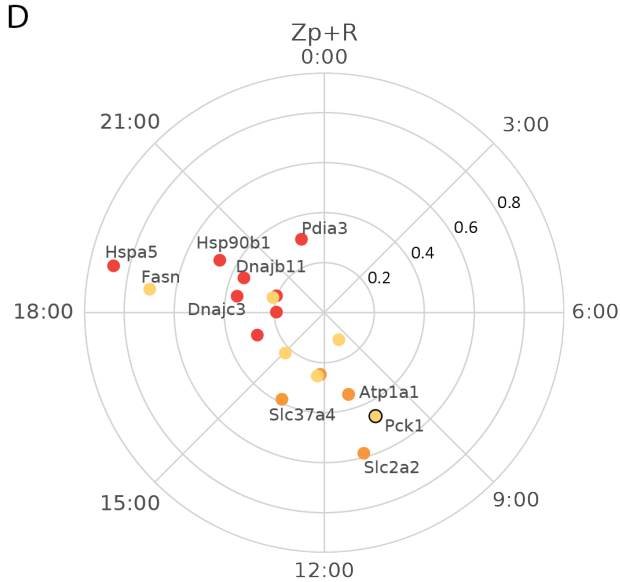

\begin{tabular}{|l|l|}
$6.15 \mathrm{E}-07$ & 61 \\
\hline
\end{tabular}

6.00E-04 85

1.11E-09 78

$1.79 \mathrm{E}-06 \quad 78$

5.98E-07 $\quad 80$

3.99E-11 83

$4.11 \mathrm{E}-05 \quad 30$

4.76E-16

$12: 00$ 00 
404 red gradient. *: appears central due to the KEGG annotation system; however, fatty acid 405 elongation is biased portally (see text).

406 (B-C-D) KEGG analysis of Z+R genes. Representations of genes in central (B-C) and 407 portal (D) enriched Z+R categories (Supplementary Table 3). Polar representation: peak 408 expression times are arranged clockwise (ZT0 on vertical position) and amplitudes (log2, 409 values indicated on the radial axes) increase radially. The radius coordinate of genes with

412 Space-time logic of activity of signaling pathways

413 Signaling pathways that include Wnt, Ras and hypoxia have been shown to shape 414 hepatocyte zonation ${ }^{8}$. We therefore examined the space-time activities of these pathways, 415 extracted from the behavior of canonical target genes. We mainly focused on Wnt, as it is 416 often considered the master regulator of liver zonation ${ }^{44}$. To systematically investigate 417 spatio-temporal WNT/ $\beta$-Catenin activity in liver, we extracted a set of Wnt targets derived 418 from an APC KO in mouse liver ${ }^{8,45}$. We found that rhythmic transcripts (in the $\mathrm{R}, \mathrm{Z}+\mathrm{R}$ and 419 ZxR categories) are enriched among targets of the Wnt pathway, showing a proportion that 420 increases with the strength of the targets, with the strongest Wnt targets containing $80 \%$ of 421 rhythmic transcripts (Supplementary Figure 7A). Positive Wnt targets were pericentral ${ }^{8}$ 422 and peaked between ZT9 and ZT12, whereas negative Wnt targets were periportal ${ }^{8}$ and 423 peaked between ZT21 and ZT3 (Figure 6A).

424 To obtain a temporal view of Wnt activity, we considered the top $50 \mathrm{Wnt}$ pathway targets 425 (according to the liver APC KO data) in the liver and analyzed the temporal profiles from 426 high temporal resolution bulk liver mRNA and from our scRNA-seq binned in three 427 different zones: central (layers 1-2), mid-lobular (layers 3-4-5) and portal (layers 6-7428 8)(Figure 6B-C, Supplementary Figure 7B-C). This analysis confirmed that the peak times 429 of rhythmic Wnt targets is preferentially between ZT9 and ZT12, and that the bulk and 430 single cell data are consistent with each other, despite of the lower temporal sampling of 431 the scRNA-seq. In fact, among the rhythmic genes detected in bulk RNA-seq, the five 432 strongest Wnt targets were, in decreasing order: Axin2, Glul, Slcla2, Tuba8, Rnf43, with 433 the latter showing the largest amplitude (Figure 6C); all but Tuba8 peaked in the morning. 434 Note that Glul, an important marker of central zonation and canonical Wnt target (just like 
435 Axin2 and Rnf43), was assigned to the $\mathrm{Z}$ category, but with second highest probability for

436 ZxR (Supplementary Table 2), peaking at ZT12. Further evidence of rhythmic WNT/ $\beta$ -

437 Catenin activity was provided by our previous proteomics data ${ }^{35}$ showing that the potent

438 Wnt effector TCF4 (encoded by the Tcf7l2 gene) has rhythmic nuclear abundance in mouse

439 liver with a peak phase at ZT7.5 (Figure 6D), and hence explains the accumulation of its

440 mRNA targets a few hours later.

441 In addition to the mRNA rhythms, we found that several of the Z+R or ZxR Wnt

442 targets showed clear rhythms in bulk proteomics with the characteristic phase delays. The

443 strongest five targets with rhythmic proteins (Supplementary Figure 7D) included the rate-

444 limiting enzyme in the bile acid biosynthetic pathway CYP7A1, the NADPH-dependent

445 monooxygenases involved in drug and xenobiotics detoxification FMO5, the P450

446 detoxification enzymes coumarin 7-hydroxylase (Cyp2a5), which may protect mice from

447 dietary coumarin-induced toxicity ${ }^{46}$, the high-affinity glutamate transporter Slc1a2

448 (Eaat2), and the multidrug resistance protein ABCC2. All these proteins showed high

449 amplitude protein rhythms peaking during the feeding phase between ZT12 and ZT18.

450 Thus, Wnt transcription activity is clearly rhythmic in the liver, and this rhythm can

451 propagate to protein expression.

452 We next asked whether the temporal oscillations in the expression of Wnt-activated

453 genes might correlate with temporal oscillations in Wnt morphogens produced by

454 pericentral non-parenchymal liver cells. To this end, we performed smFISH experiments

455 and quantified the expression of the Wnt ligand $W n t 2^{5}$ and of $R s p o 3^{6,47}$, a critical facilitator

456 of Wnt signaling, as well as the Wnt antagonist $D k k 3^{19}$ (Figure 6E). We found that both

457 Wnt2 and Rspo3 in liver non-parenchymal cells (NPCs) exhibit non-uniform expression

458 around the clock, with significantly higher mRNA levels at ZT0 ( $\mathrm{p}=4 \times 10^{-5}$ for $W n t 2$, and

$459 \mathrm{p}=2 \times 10^{-8}$ for Rspo3, Kruskal-Wallis, Figure 6E, right). Given various delays between

460 mRNA accumulation of ligands and expression of the Wnt targets, this timing is

461 compatible with the peak nuclear accumulation of the TCF4 (Tcf712 gene) transcription

462 factor observed at ZT7.5 (Figure 6D) and with the peaks in Wnt-activated genes between

463 ZT6-12 (Figure 6A-B). Differences in Dkk3 expression were not significant $(\mathrm{p}=0.053)$.

464 Thus, production of Wnt morphogens by central non-parenchymal liver cells might

465 underlie the observed rhythmic Wnt pathway activity. 
Figure 6. Rhythmic activity of Wnt signaling.

A

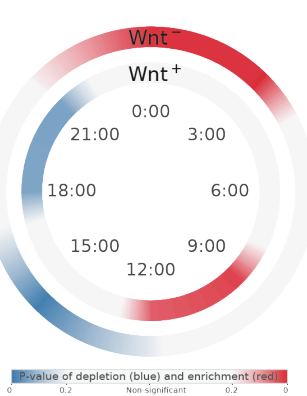

B Central Middle Portal
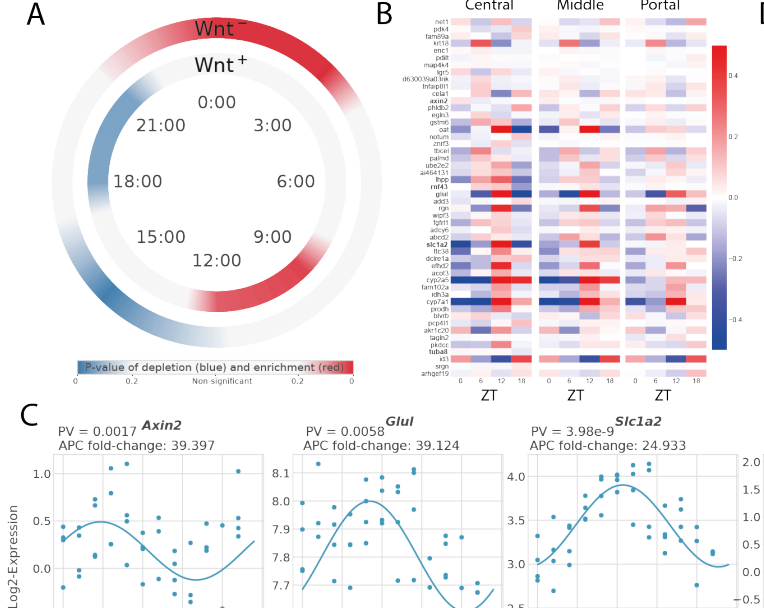

D

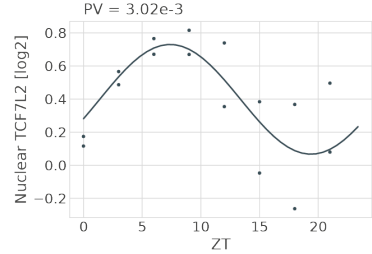

$\mathrm{PV}=1.48 \mathrm{e}-\mathrm{T}^{\text {Tubas }}$
APC fold-change: 23.588

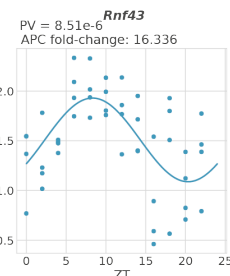

central: $\mathrm{PV}=4.58 \mathrm{e}-01$
middle: $\mathrm{PV}=7.70 \mathrm{e}-01$
portal $\mathrm{PV}=1.13 \mathrm{e}+00$

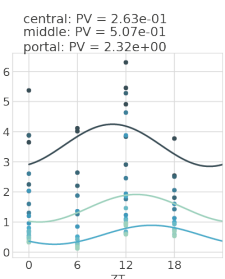

- Fit portal profile

$\mathrm{E}$
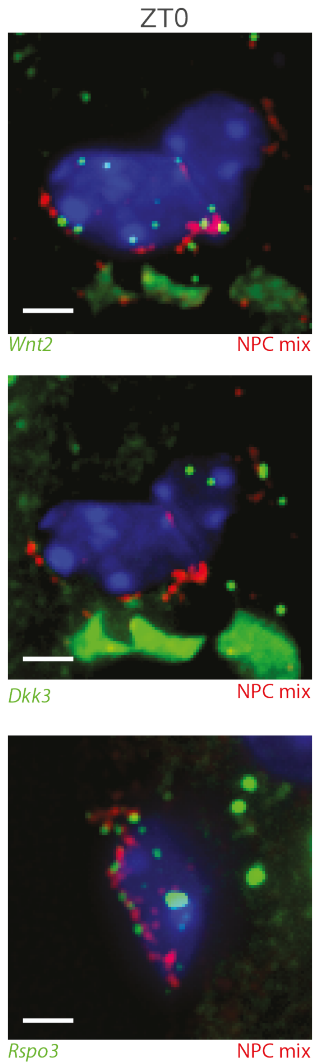

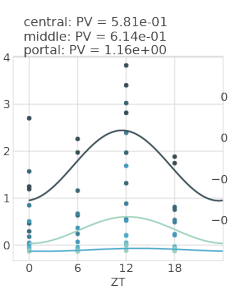

- Fit mid profile

ZT18
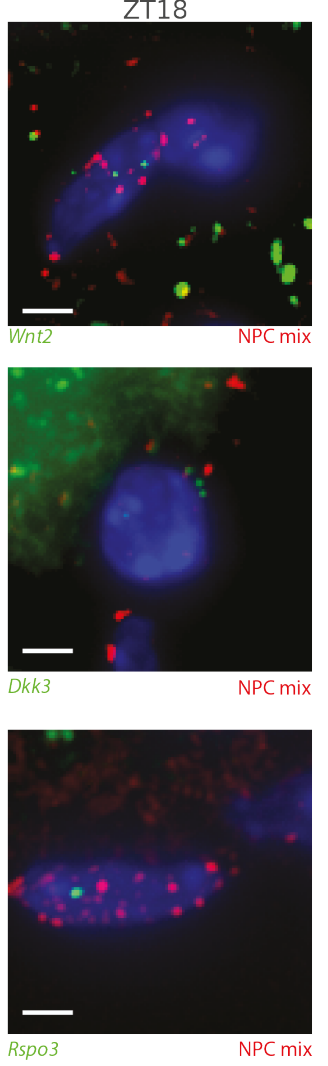

central : $P V=4.892-01$
middal $P V=5.86-01$
portal: $P V=1.48 \mathrm{e}+00$

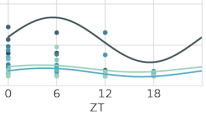

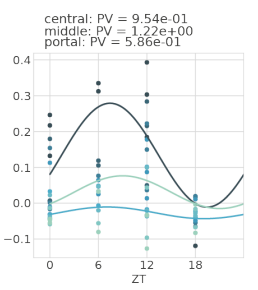

$x=7 \quad x=8$

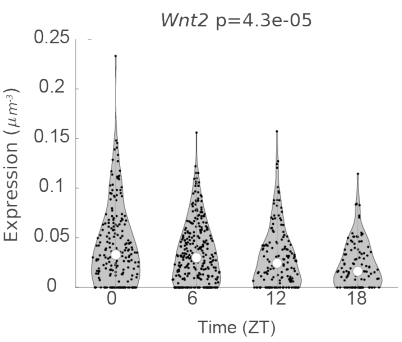

$D k k 3 \mathrm{p}=0.053$

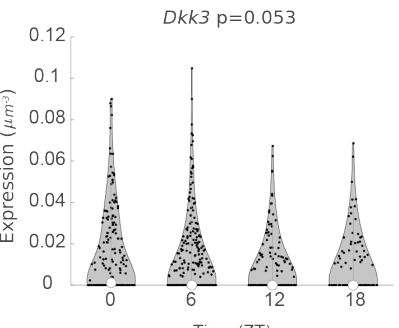

Rspo3 $\mathrm{p}=1.5 \mathrm{e}-08$

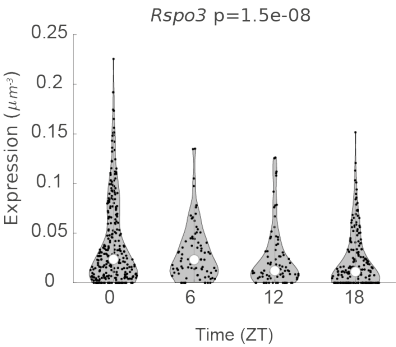


468 (A) Enrichment/depletion at different times (window size: 3h), of both positive and 469 negative Wnt targets (background: all R and Z+R genes). Colormap shows p-values (two470 tailed hypergeometric test): red (blue) indicates enrichment (depletion).

(B) Heatmaps representing scRNA-seq profiles of the top $50 \mathrm{Wnt}$ targets (according to the Apc-KO fold change, $\operatorname{Lgr} 5$ was also added) showing rhythmic mRNA in bulk $(\mathrm{p}<0.01$, ref. $\left.{ }^{25}\right)$. The profiles are computed in three different zones of the central-portal axis: central (layers 1-2), mid-lobular (layers 3-4-5) and portal (layers 6-7-8). Gene profiles ( $\log 2)$ are mean-centered in each zone. An enrichment of the phases around ZT8-14 can be observed, in agreement with Figure 6A.

(C) mRNA profiles from bulk RNA-seq (top) and scRNA-seq (bottom). Top five targets with the highest Apc-KO fold change, and rhythmicity in the bulk data $(\mathrm{p}<0.01$, harmonic regression). Rhythmicity (indicated above each panel) is computed on bulk data or in three different zones for scRNA-seq data.

(D) Nuclear protein abundance from ref. ${ }^{35}$ of the Wnt effector TCF4 (encoded by the Tcf7l2 gene) in mouse liver shows a rhythm $(p=0.003)$ peaking at ZT7.5, consistent with the accumulation of mRNA targets a few hours later (panel A).

(E) Left: Representative smFISH images of $W n t 2, D k k 3$, and Rspo3 expression at ZT0 (left) and ZT18 (right), shown in green. Markers of non-parenchymal cells (NPCs) are shown in red (Methods). Nuclei are stained in blue (DAPI). Scale bars, $2 \mu \mathrm{m}$; Right: Violin plots representing quantitative analysis of smFISH images (1420 cells of 189 central veins of at least two mice per time point). Wnt2, Dkk3, and Rspo3 transcripts are quantified in NPCs lining the central vein (Methods). mRNA expression is in smFISH dots per $\mu \mathrm{m}^{3}$.

492 of Ras were enriched in central genes, whereas the positive Ras targets were enriched in 493 portal genes (not shown). The rhythmic targets ( $R$ and $\mathrm{Z}+\mathrm{R}$ ) of hypoxia showed a pattern 494 of temporal compartmentalization similar with those of Wnt (Supplementary Figure 7E): 495 the negative targets were enriched around ZT0 (dark-light transition) and underrepresented 496 around ZT14, while the positive targets were enriched around ZT10 and underrepresented 497 around ZT3. Ras targets, positive or negative, did not exhibit significant temporal bias. 


\section{Discussion}

Recent genome-wide analyses of zonated gene expression in mouse and human

500 liver ${ }^{8,48,49}$ uncovered a rich organization of liver functions in space at the sublobular scale,

501 while chronobiology studies of bulk liver tissue revealed a complex landscape of rhythmic

502 regulatory layers orchestrated by a circadian clock interacting with feeding-fasting cycles

503 and systemic signals ${ }^{35,50-52}$. Here, we established how these two regulatory programs

504 combine to shape the daily space-time dynamics of gene expression patterns and

505 physiology in adult liver by extending our previous scRNA-seq approach ${ }^{8}$. We found that

506 liver uses gene expression programs with many genes exhibiting compartmentalization in

507 both space and time.

508 In this study, we chose to focus on the parenchymal cells in the liver, the 509 hepatocytes, for which smFISH data on landmark zonated genes was readily available, 510 which enabled reconstructing spatio-temporal mRNA profiles from scRNA-seq ${ }^{8}$. Zonation 511 profiles of other cell types in the liver may be obtained as well; in fact, static zonation 512 mRNA expression profiles have been obtained for liver endothelial cells, using a paired513 cell approach ${ }^{19}$ in which mRNA from pairs of attached mouse cells were sequenced and 514 gene expression from one cell type was used to infer the pairs' tissue coordinates. In 515 addition, ab initio reconstruction methods such as diffusion pseudo time ${ }^{48}$ or novoSpaRc ${ }^{53}$,

516 in which a zonation coordinate is inferred by assuming that the major axis of variability for 517 a cell type reflects transcriptome-wide gene expression changes associated with zonation, 518 could be used for spatially sparse cell types with no available zonated marker genes, e.g. 519 stellate or resident immune Kupffer cells. Moreover, it was recently found that rhythmic 520 gene expression and metabolism in non-hepatocyte cells can be driven both by clocks in 521 hepatocytes via cell-cell communication as well as feeding cycles ${ }^{13}$. Our computational

522 framework for analyzing space-time logic of gene expression could be widely applicable 523 in such future studies.

$524 \quad$ To study whether the observed space-time expression profiles may be regulated by 525 either liver zonation, $24 \mathrm{~h}$ rhythms in liver physiology, or both, we developed a mixed526 effect model, combined with model selection. This enabled classifying gene profiles into 527 five categories representing different modes of spatio-temporal regulation, from flat to 528 wave-like. To validate these, we performed smFISH in intact liver tissue, which showed 
529 largely compatible profiles although some quantitative differences were observed. These

530 differences most likely reflect the lower sensitivity of RNA-seq, uncertainties in the spatial

531 analysis of smFISH in tissues, as well as known inter-animal variability in the physiologic

532 states of individual livers, notably related to the animal-specific feeding patterns ${ }^{25}$.

533 Together, this temporal analysis confirms that a large proportion of gene expression

534 in hepatocytes is zonated ${ }^{8}$ or rhythmic $^{17}$, and in addition reveals marked spatio-temporal

535 regulation of mRNA levels in mouse liver $(\mathrm{Z}+\mathrm{R}$ and $\mathrm{ZxR}$ genes, comprising $7 \%$ of all

536 detected genes according to our criteria). This means that zonated gene expression patterns

537 can be temporally modulated on a circadian scale, or equivalently, that rhythmic gene

538 expression profiles can exhibit sub-lobular structure. The dominant pattern for dually

539 regulated gene was $Z+R$, which corresponds to additive effects of space and time in $\log$, or

540 multiplicate effects of gene expression levels, and describes genes expression profiles that

541 are compartmentalized in both space and time. In other words, such patterns are

542 characterized by shapes (in space) that remain invariant with time, but whose magnitudes

543 are rhythmically rescaled in time. Or equivalently, the oscillatory amplitude (fold change)

544 and phases are constant along the lobular coordinate, but the mean expression is patterned

545 along the lobule. Such multiplicative effects could reflect the combined actions of

546 transcriptional regulators for the zone and rhythm on promoters and enhancers of $\mathrm{Z}+\mathrm{R}$

547 genes. Indeed, gene expression changes induced by several regulators combine

548 multiplicatively ${ }^{22}$. Note that though the (relative) shape of $Z+R$ patterns is invariant in time,

549 threshold-dependent responses that would lie downstream of such genes would then

550 acquire domain boundaries which can shift in time. Similar phenomena are expected for

551 interacting profiles (phase and amplitude modulated) (ZxR) that we observed for a smaller

552 number of genes.

553 As shown by us and others ${ }^{27,28}$, rhythms at the protein level are typically damped

554 and phase-delayed compared the cognate mRNA rhythms, depending on the protein half-

555 lives. Indeed, longer protein half-lives imply smaller oscillatory amplitudes and longer

556 delays between mRNA and protein accumulation ${ }^{14}$. Analysis of liver proteomes in bulk

557 showed that the number of cyclic proteins is significantly lower than the number of cyclic

558 mRNAs, with delays approaching the predicted maximum of six hours. Here, we found

559 genes from both $\mathrm{Z}+\mathrm{R}$ and $\mathrm{ZxR}$ that exhibited rhythmic accumulation in bulk proteomics 
560 experiments, including genes encoding rate-limiting enzymes, suggesting that dually 561 space-time regulated patterns have a physiological role in the liver. Moreover, phase delays

562 between the mRNA and protein profiles were as expected. Future studies will utilize 563 emerging spatial proteomics approaches to reconstruct a space-time liver proteomic atlas ${ }^{54}$. 564 In addition to previously discussed zonated liver functions ${ }^{8}$, a systematic querying 565 of KEGG pathways highlighted $\mathrm{Z}+\mathrm{R}$ functions not previously associated with rhythmic 566 liver zonation. The roles and profiles of the corresponding genes allowed us to better 567 understand the spatiotemporal logic of the identified pathways. For instance, we found that 568 the expression levels of both ribosome protein genes (rate-limiting for protein synthesis ${ }^{32}$ ) 569 and proteasome components (involved in protein degradation) were higher in central 570 hepatocytes. Since the central environment is subject to high concentrations of xenobiotics 571 and hypoxic stress, this could indicate an elevated protein turnover in this region, which 572 would ensure that damaged proteins are rapidly exchanged with new, undamaged proteins. 573 This interpretation is corroborated by the observed increased levels of cytosolic and ER 574 chaperones during the feeding phase, to assist protein synthesis and secretion, thereby 575 counteracting such protein stress.

576 It was previously shown that Wnt signaling can explain the zonation of up to a third 577 of the zonated mRNAs ${ }^{7}$. Wnt ligands are secreted by pericentral non-parenchymal cells, 578 mostly endothelial cells ${ }^{5,6}$, forming a graded spatial morphogenetic field. As a result, and 579 as observed in our enrichment analysis, Wnt-activated genes were pericentrally-zonated. 580 Moreover, both the scRNA-seq data and previous bulk mRNA and protein measurements 581 showed that Wnt activity is rhythmic in the liver. Our smFISH analysis suggested that 582 temporal fluctuations in the expression of Wnt2 and Rspo3, two key Wnt ligands, secreted 583 by pericentral non-parenchymal cells might underlie oscillatory and zonated expression of 584 Wnt targets at times near the fasting/feeding transition.

585 In summary, we demonstrate how liver gene expression can be quantitatively 586 investigated with spatial and temporal resolution and how liver functions are 587 compartmentalized along these two axes. Our approach could be used to reconstruct spatio588 temporal gene expression patterns in other zonated tissues such as the intestine and kidney ${ }^{3}$. 
590 Material and methods

$591 \quad$ Animals and ethics statement

592 All animal care and handling were approved by the Institutional Animal Care and Use 593 Committee of WIS and by the Canton de Vaud laws for animal protection (authorization 594 VD3197.b). Male C57b16 mice aged of 6 weeks, housed under reverse-phase cycle and 595 under ad libitum feeding were used to generate sc-RNA-seq data of hepatocytes and single596 molecule RNA-FISH (smFISH). Male mice between 8 to 10 weeks old, housed under 12:12 597 light-dark cycle, and having access to food only during the night (restricted-feeding) were 598 used for smFISH of circadian clock genes.

601 Liver cells were isolated using a modified version of the two-step collagenase perfusion 602 method of Seglen ${ }^{55}$. The tissue was digested with Liberase Blendzyme 3 recombinant 603 collagenase (Roche Diagnostics) according to the manufacturer instructions. To enrich for 604 hepatocytes, we applied a centrifuge step at $30 \mathrm{~g}$ for $3 \mathrm{~min}$ to pull down all hepatocytes 605 while discarding most of the non-parenchymal cells that remained in the sup. We next 606 enriched for live hepatocytes by 2 cycles of percoll gradient, hepatocytes pellet was 607 resuspended in $25 \mathrm{ml}$ of PBS, percoll was added for a final concentration of $45 \%$ and mixed 608 with the hepatocytes. Dead cells were discarded after a centrifuge step (70g for $10 \mathrm{~min})$ 609 cells were resuspended in 10x cells buffer (1x PBS, 0.04\% BSA) and proceeded directly 610 to the $10 \mathrm{x}$ pipeline. The cDNA library was prepared with the V2 chemistry of $10 \mathrm{X}$ 611 genomics Chromium system according to manufactures instructions and sequencing was 612 done with Illumina Nextseq 500 at estimated depth of 40,000 reads per cell.

614 Conceivably, the dissociation of liver tissue into individual cells and the purification of 615 hepatocytes are relatively lengthy and may thus lead to alterations in mRNA expression. 616 While it has been shown that mRNA levels do not change much during the purification and 617 24-hour cultivation of hepatocytes ${ }^{56}$, transcription rates on the other hand can be 618 diminished by 8 -fold to nearly 100 -fold during this process ${ }^{57}$. This difference between 619 nascent transcript and mature mRNA levels can be explained by the relatively long half620 lives of liver-specific RNAs. In our case, the time needed from the dissociation of the tissue 
621 until the cell lysis is approximately 1 hour and the cell are not placed in culture. Since we

622 are measuring mature transcripts, with half-lives in the range of typically 1-5 hours

623 (Supplementary Figure 2C), the changes in mRNA levels due to the protocol will remain

624 contained. In particular, the scRNA-seq of cells carry the typical hepatocyte gene

625 expression signatures, for example, genes such as Alb or Apoa2 rank at 2nd and 5th

626 position genome-wide. As further validation, we compared our reconstructed gene

627 expression zonation profiles with the zonation profiles from the massively validated

628 zonation study of ref. $^{8}$, revealing a near-perfect agreement (Supplementary Figure 1F).

\section{Filtering of raw scRNA-seq data}

631 The initial data analysis was done in R v3.4.2 using Seurat v2.1.0 ${ }^{58}$. Each expression matrix

632 was filtered separately to remove dead, dying and low-quality cells. We firstly only kept

633 genes that were expressed in at least 5 cells in any of the ten samples. We then defined a

634 set of valid cells with more than 500 expressed genes and between 1000 and 10000 unique

635 molecular identifiers (UMIs) and secondly an additional expression matrix with cells

636 having between 100 and 300 UMIs which was used for background estimation

637 (Supplementary Figure 1A). Other UMI-filters have been tried, but yielded equal or less

638 reliable profiles. The mean expression of each gene was then calculated for the background

639 dataset and subtracted from the set of valid cells. This was subsequently filtered to only

640 include hepatocytes by removing cells with expression of non-parenchymal liver cell

641 genes. Next, the cells were filtered based on the fraction of mitochondrial gene expression.

642 First, expression levels in each cell were normalized by the sum of all genes excluding

643 mitochondrial and major urinary protein (Mup) genes. Indeed, as mitochondria are more

644 abundant in periportal hepatocytes, the expression of mitochondrial genes is higher in this

645 area ${ }^{59}$; and since these genes are very highly expressed, including them would reduce the

646 relative expression of all other genes based on the cell's lobular location. Mup genes are

647 also highly abundant and mapping their reads to a reference sequence is unreliable due to

648 their high sequence homology ${ }^{60}$. Moreover, Mup genes encode for pheromones that vary

649 greatly between individuals to facilitate individual recognition ${ }^{61}$.

650 Mitochondrial content is often used to remove non-viable cells ${ }^{62}$. The mitochondrial 651 content of sequenced hepatocytes exhibited a bi-modal behavior (Supplementary Figure 
652 1B). To identify the range of mitochondrial fractions that included viable hepatocytes we 653 used an intrinsic property of hepatocytes, which is the anti-correlation of the pericentral

654 landmark gene Cyp2el and the periportal landmark gene Cyp $2 f 2^{7}$ (Supplementary Figure 655 1C). We found that hepatocytes with mitochondrial fraction in the range of 9-35\% 656 exhibited an almost perfect anticorrelation between Cyp2e1 and Cyp2f2 (Supplementary 657 Figure 1D,E), suggesting that these are the best quality, and we consequently kept 658 hepatocytes within this range of mitochondrial content for further analysis.

661 To validate that the expected spatial and temporal axes of variation are present in the 662 scRNA-seq data, we generated a low-dimensional representation of all cells using the 663 standard t-SNE (t-distributed stochastic neighbor embedding) ${ }^{63}$, a nonlinear dimensionality 664 reduction technique that embeds high-dimensional data on a 2-dimensional plane such that 665 points that are similar in high-dimensional space are close together on the 2-dimensional 666 representation. We then colored cells either by their position along the central-portal axis, 667 or by time of day.

669 Spatial reconstruction of zonation profiles from scRNA-seq data

670 Choice of landmark genes

671 The reconstruction algorithm relies on a priori knowledge about the zonation of a small 672 set of landmark genes to infer the location of the cells. Ref. ${ }^{8}$ used smFISH to determine the 673 zonation pattern in situ of 6 such landmark genes and used them to reconstruct the spatial 674 profiles of all other genes at a single time point. Since we here aimed at reconstructing 675 zonation profiles at different time points, we could not rely on those landmark genes, which

676 might be subject to temporal regulation. Therefore, we used an alternative strategy where 677 we selected landmark zonated genes from ref. ${ }^{8}(\mathrm{q}<0.2)$, with the additional constraints 678 that those should be highly expressed (mean expression in fraction UMI of more than $6790.01 \%$ and less than $0.1 \%$ ), and importantly vary little across mice and time. Specifically, 680 we calculated the variability in the mean expression (across all layers) between all mice for 681 every gene and removed genes with $>=10 \%$ variability. This yielded 27 central (Akr $1 c 6$, 682 Alad, Blvrb, C6, Car3, Ccdc107, Cml2, Cyp2c68, Cyp2d9, Cyp3a11, Entpd5, Fmo1, 
683 Gsta3, Gstm1, Gstm6, Gstt1, Hpd, Hsd17b10, Inmt, Iqgap2, Mgst1, Nrn1, Pex11a, Pon1,

684 Psmd4, Slc22a1, Tex264); and 28 portal (Afm, Aldh1l1, Asl, Ass1, Atp5a1, Atp5g1, C8a,

685 C8b, Ces3b, Cyp2f2, Elovl2, Fads1, Fbp1, Ftcd, Gm2a, Hpx, Hsd17b13, Ifitm3, Igf1,

686 Igfals, Khk, Mug2, Pygl, Sepp1, Serpinalc, Serpinale, Serpind1, Vtn) landmark genes.

Reconstruction algorithm

689 The reconstruction algorithm is based on the algorithm in ref. ${ }^{8}$ and was used in the modified

690 version from ref. ${ }^{19}$. The procedure was applied independently on each mouse, yielding ten

691 spatial gene expression profiles for each gene, given as fraction of UMI per cell.

Spatiotemporal analysis of liver gene expression profiles

694 Data

695 Each profile for the 14678 genes includes 8 layers from the pericentral to the periportal 696 zone and 4 time points: ZT0 (n=3 biological replicates from individual mice), ZT6 ( $\mathrm{n}=2$ ),

697 ZT12 $(\mathrm{n}=3)$ and ZT18 $(\mathrm{n}=2)$. The expression levels (noted as $x$ ) are then log-transformed 698 as follows:

$$
y=\log _{2}(x+\Delta)-B
$$

700 The offset $\Delta=10^{-4}$ buffers variability in lowly expressed genes, while the shift $B=$

$701-\log _{2}\left(11 \times 10^{-5}\right)$ changes the scale so that $y=0$ corresponds to about $10 \mathrm{mRNA}$ copies

702 per cell (we expect on the order of 1M mRNA transcripts per liver cell).

704 Reference genes

705 For ease of interpretation (Figure 2 and Supplementary Figure 2), we used a set of reference

706 circadian genes and a set of reference zonated genes, highlighted in several figures.

707 The reference core circadian clock and clock output genes are the following: Bmall, Clock,

708 Npas2, Nr1d1, Nr1d2, Per1, Per2, Cry1, Cry2, Dbp, Tef, Hlf, Elovl3, Rora, Rorc.

709 The reference zonated genes are the following: Glul, Ass1, Asl, Cyp2f2, Cypla2, Pck1,

710 Cyp2e1, Cdh2, Cdh1, Cyp7a1, Acly, Alb, Oat, Aldob, Cps1. 
713 To analyze variability in space and time (Figure 2A) we computed, for each gene, the

714 spatial variance $V_{x}$ and the temporal variance $V_{T}$. Let $y_{x, t, j}$ represent the expression profile,

715 with $j$ the replicate index, $t \in\left\{1,2, \ldots, N_{t}\right\}$ the time index, and $x \in\left\{1,2, \ldots, N_{x}\right\}$ the layer

716 index. Then, $V_{x}$ and $V_{T}$ are computed as follows:

$$
V_{X}=\frac{1}{N_{t}} \sum_{t} \frac{\sum_{x}\left[\sum_{j}\left(y_{x, t, j}-\frac{1}{N_{x}} \sum_{x} y_{x, t, j}\right)\right]^{2}}{N_{j}^{2} N_{x}}
$$

$$
V_{T}=\frac{1}{N_{x}} \sum_{x} \frac{\sum_{t}\left[\sum_{j}\left(y_{x, t, j}-\frac{1}{N_{t}} \sum_{t} y_{x, t, j}\right)\right]^{2}}{N_{j}^{2} N_{t}}
$$

718 Thus, the spatial variance $V_{x}$ is computed along the space (and averaged over the replicates)

719 for each time condition, and then averaged over time. The procedure is similar, 720 symmetrically, for $V_{t}$.

Gene filtering

723 For the analyses in Figure 2, we selected transcripts that were reproducible between

724 replicates, as well as sufficiently highly expressed (see scatterplot in Supplementary Figure

725 2A). To assess reproducibility across replicates, we computed the average relative variance

726 of the spatiotemporal profiles over the replicates:

$$
V_{J}=\frac{\frac{1}{N_{x} N_{t}} \sum_{x, t}\left[\frac{1}{N_{j}} \sum_{j}\left(y_{x, t, j}-\frac{1}{N_{j}} \sum_{j} y_{x, t, j}\right)^{2}\right]}{\frac{1}{N_{x} N_{t} N_{j}} \sum_{x, t, j}\left[\left(y_{x, t, j}-\frac{1}{N_{x} N_{t} N_{j}} \sum_{x, t, j} y_{x, t, j}\right)^{2}\right]}
$$

728 We considered genes with values below 50\% (Supplementary Figure 2). To filter lowly

729 expressed genes, we required the maximum expression level across layers and time points

730 to exceed $10^{-5}$ (fraction of UMIs) which corresponds to $y=0$ or about 10 copies of mRNA

731 per cell. While this was quite more permissive than previous scRNA-seq studies it allowed

732 to keep most reference circadian and zonated genes. However, scRNA-seq has still limited

733 sensitivity and some potentially important genes may have been removed in the filtering

734 process. In the end, our filters kept 5085 genes (1437 were removed due to low expression,

7354733 due to high variance, and 3543 due to both), which were then used for subsequent 736 analyses. 
Mixed-effect model for spatiotemporal $m R N A$ profiles

739 Since the data is longitudinal is space (8 layers measured in each animal), modelling the

740 space-time profiles require the use of mixed-effect models. To systematically analyze the

741 spatiotemporal mRNA profiles, we used a parameterized function. Specifically, the model

742 uses sine and cosine functions for the time, and polynomials (up to degree 2) for space.

743 Possible interaction between space and time are described as space-dependent oscillatory

744 functions, or equivalently, time-dependent polynomial parameters. Our model for the

745 transformed mRNA expression $y$ reads:

$$
y_{x, t, i}=\mu_{i}+\mu(x)+a(x) \cos (\omega t)+b(x) \sin (\omega t)+\varepsilon_{x, t, \mathrm{i}}
$$

747 Here $t$ is the time, $x$ the spatial position along the liver layers, and $i \in\{1,2, \ldots, 10\}$ the 748 animal index. This function naturally generalizes harmonic regression, often used for 749 analysis of circadian gene expression ${ }^{25}$, by introducing space-dependent coefficients:

$$
\left\{\begin{array}{l}
\mu(x)=\mu_{0}+\mu_{1} P_{1}(x)+\mu_{2} P_{2}(x) \\
a(x)=a_{0}+a_{1} P_{1}(x)+a_{2} P_{2}(x) \\
b(x)=b_{0}+b_{1} P_{1}(x)+b_{2} P_{2}(x)
\end{array}\right.
$$

751 Here, $P_{1}$ and $P_{2}$ are the Legendre polynomials of degrees 1 and 2, respectively; $\mu_{0}, \mu_{1}$ and $752 \mu_{2}$ represent the static zonation profile, $a_{0}$ and $b_{0}$ represent the global (space-independent) 753 rhythmicity of the gene, while $a_{1}, a_{2}, b_{1}, b_{2}$ represent layer-dependent rhythmicity. $\varepsilon_{x, t, j}$

754 is a Gaussian noise term with standard deviation $\sigma$. In addition to the fixed-effect 755 parameters described so far, we also introduced a mouse-specific random-effect $\mu_{i}$ (with 756 zero mean). This parameter groups the dependent layer measurements (obtained in the 757 same animal) and thereby properly adjusts the biological sample size for the rhythmicity 758 analysis.

759 Phases $\varphi$ (related to peak times $t$ through $t=\varphi * 24 / 2 \pi$ ) and amplitudes $A$, for each 760 profile can then be computed for any layer from the coefficients $a(x)$ and $b(x)$ :

$$
\varphi(x)=\arctan 2(b(x), a(x)) \quad A(x)=\sqrt{a(x)^{2}+b(x)^{2}}
$$

762 Note that peak-to-trough difference is $2 A(x)$. The peak-to-trough ratio or fold change of

763 the original expression levels is then $2^{2 A(x)}$. We also note that an equivalent writing of the 764 model formulates the problem in terms of time-dependent zonation parameters instead of 765 space-dependent rhythmicity:

$$
y_{x, t, i}=\mu_{i}+\mu_{0}(t)+\mu_{1}(t) P_{1}(x)+\mu_{2}(t) P_{2}(x)+\varepsilon_{x, t, i}
$$


767 where:

$$
\left\{\begin{array}{l}
\mu_{0}(t)=\mu_{0}+a_{0} \cos (\omega t)+b_{0} \sin (\omega t) \\
\mu_{1}(t)=\mu_{1}+a_{1} \cos (\omega t)+b_{1} \sin (\omega t) \\
\mu_{2}(t)=\mu_{2}+a_{2} \cos (\omega t)+b_{2} \sin (\omega t)
\end{array}\right.
$$

769 In this study, we fixed $\omega=\frac{2 \pi}{24 h}$ since the animals were entrained in a $24 \mathrm{~h}$ light-dark cycle

770 and the low time resolution would prevent us from studying ultradian rhythms.

771 The model parameters, including the variance of the random effects and Gaussian noise

772 strength $\sigma$, are estimated for each gene using the fit function from the Python library

773 StatsModels (version 0.9.0). Nelder-Mead was chosen as the optimization method, and the

774 use of a standard likelihood was favored over the REML likelihood to allow for model

775 comparison ${ }^{64}$. To prevent overfitting of the gene profiles, we added a noise offset $\sigma_{0}=0.15$

$776\left[\log _{2}\right]$ to the estimated noise $\sigma$, in the expression of the likelihood function used in the

777 mixed-effect model optimization.

779 Depending on the gene, the model presented in $(v)$ and (vii) may be simplified by setting 780 all or some of the (fixed) parameters to 0. For example, a non-oscillatory gene profile 781 would normally have non-significant $a_{j}$ and $b_{j}$ parameters. In practice, considering the 782 fixed effects, $2^{9}$ sub-models of various complexity can be generated. However, we added 783 a few reasonable requirements to reduce the number of models. First, the intercept $\mu_{0}$ must 784 be present in every model. Similarly, the parameters $a_{0}$ and $b_{0}$, providing a global rhythm, 785 must be present in every rhythmic model. Finally, the parameters $a_{j}$ and $b_{j}$ for $j=0,1,2$ must 786 be paired to ensure a proper phase definition ( $v i)$.

787 The models can then be classified in different categories, depending on the retained (non788 zero) parameters (Figure 2C):

789 - The model comprising only the intercepts $\mu_{0}$ and $\mu_{i}$, termed flat or noisy $(\mathrm{F} / \mathrm{N})$.

790 - The models comprising only the intercepts and zonation parameters: $\mu_{1}$ and/or $\mu_{2}$, 791 termed purely zonated $(Z)$.

792 - The models comprising only the intercepts and rhythmic parameters: $a_{0}$ and $b_{0}$, 793 termed purely rhythmic (R).

794 - The models comprising only the intercepts, zonated parameters and rhythmic 795 parameters: $\mu_{1}$ and/or $\mu_{2}$, and $a_{0}, b_{0}$, termed independent $(\mathrm{Z}+\mathrm{R})$. 
- The models comprising interaction parameters: $a_{j}$ and $b_{j}$ for $j=1,2$, termed interacting $(\mathrm{ZxR})$.

798 Note that we only plot the fixed effects in the predicted gene profiles. The Bayesian

799 Information Criterion (BIC) is then used for model selection, enabling to choose the most

800 parsimonious model for each gene. Consequently, the $\mathrm{F} / \mathrm{N}$ class also contains noisy

801 profiles, since genes that are not well fitted with any complex model will then be assigned

802 to the simplest model. Additionally, it appears that, for some profiles, several competing

803 models can result in close BIC values (see e.g. the discussion on Clock and Cry1 in the

804 Results). Therefore, when assigning hard classes, if some models have a relative difference

805 of less than $1 \%$ in their BIC, we systematically keep the most complex model. Moreover,

806 we also assigned probabilities to the different categories (F, Z, R, Z+R and ZxR), computed

807 as Schwartz BIC weights ${ }^{65}$, which is useful in case of ambiguous classification

808 (Supplementary Table 2). All best fits with their parameter values are listed in

809 Supplementary Table 1.

811 Comparison of peak times with a bulk dataset

812 We compared the peak phases our rhythmically classified genes with those obtained from

813 ref. ${ }^{25}$. These data consist of bulk liver RNA-seq data sampled every 2 hours for 24 hours,

814 with 4 replicates per time condition. We only compared genes that for which rhythmicity

815 is not changing across layers, viz. the $\mathrm{R}$ and $\mathrm{Z}+\mathrm{R}$ categories. Note that since our dataset has

816 a lower temporal resolution and fewer replicates per time point, we found overall less

817 rhythmic genes.

818 To assess gene rhythmicity from ref. ${ }^{25}$, we used harmonic regression on the log-

819 transformed profiles as previously. Using the same notation as above, we define the two

820 following models:

$$
\left\{\begin{array}{lll}
y_{t, i} & =\mu+\varepsilon \\
y_{t, i} & =\mu+a \cos (\omega t)+b \sin (\omega t)+\varepsilon & (x)
\end{array}\right.
$$

822 We then fit eq. (viii) and eq. (ix) to every transcript, and, for each of them, keep the model

823 with the lowest BIC. We then compared the phases of transcripts classified as rhythmic in

824 both datasets, and computed the circular correlation coefficient ${ }^{66}$. 


\section{KEGG pathway Enrichment analysis}

827 Functional annotation clustering from Enrichr ${ }^{67}$ for the categories $\mathrm{F} / \mathrm{N}, \mathrm{Z}$ and $\mathrm{Z}+\mathrm{R}$ (which

828 is then subdivided in central and portal), $\mathrm{Zc}+\mathrm{R}$ (central zonated and rhythmic), $\mathrm{Zp}+\mathrm{R}$

829 (portal zonated and rhythmic) and finally $\mathrm{R}$ was ran with standard parameters, using the

830 standard KEGG 2019 Mouse set of pathways. The enriched pathways (adjusted p-value <

831 0.1) was then further annotated to compute, e.g. the number of central/portal genes in each

832 category and the phase of each gene. This analysis is available Supplementary Table 3.

\section{4 smFISH}

835 Analysis of $Z+R$ and $Z x R$ genes (Stellaris smFIH probes)

836 Preparation of probe libraries, hybridization procedure and imaging conditions were 837 previously described ${ }^{19}$. smFISH probe libraries were coupled to TMR, Alexa594 or Cy5. 838 Cell membranes were stained with alexa fluor 488 conjugated phalloidin (Rhenium 839 A12379) that was added to GLOX buffer $^{68}$. Portal node was identified morphologically on 840 DAPI images based on bile ductile, central vein was identified using smFISH for Glul in 841 TMR, included in all hybridizations. Images were taken as scans spanning the portal node

842 to the central vein. Images were analyzed using Image ${ }^{68}$. Quantification of zonation 843 profiles in different circadian time point were generated by counting dots and dividing the 844 number of dots in radial layers spanning the portal-central axis by the layer volume.

846 Temporal analysis of circadian genes (RNA scope smFISH probes)

847 smFISH of R genes were done on fresh-frozen liver cryosections $(8 \mu \mathrm{m})$ embedded in 848 O.C.T Compound (Tissue-Tek; Sakura-Finetek USA), sampled every three hours (ZT0 to 849 ZT21). RNAscope ${ }^{\circledR}$ probes for Bmall mRNA (Mm-Arntl, catalog \#: 438748-C3) and Perl 850 mRNA (Mm-Per1, catalog \#: 438751) were used, according to the manufacturer's 851 instructions for the RNAscope Fluorescent Multiplex V1 Assay (Advanced Cell 852 Diagnostics). To detect the central vein, an immunofluorescence of Glutamine Synthetase 853 (ab49873, Abcam, diluted 1:2000 in PBS/BSA 0.5\%/Triton-X0.01\%) was done together 854 with smFISH. Nuclei were counterstained with DAPI and sections were mounted with 855 ProLong ${ }^{\text {TM }}$ Gold Antifade Mountant. Liver sections were imaged with a Leica DM5500 856 widefield microscope and an oil-immersion x63 objective. Z-stacks were acquired $(0.2 \mu \mathrm{m}$ 
857 between each $\mathrm{Z}$ position) and mRNA transcripts were quantified using ImageJ, as

858 described previously in ref. ${ }^{50}$. Pericentral (PC) and Periportal (PP) veins were manually

859 detected based on Glutamine Synthetase IF or on bile ducts (DAPI staining). The Euclidean

860 distance between two veins and the distance from the vein of each mRNA transcript were

861 calculated. mRNA transcripts were assigned to a PP or PC zone if the distance from the

862 corresponding vein was smaller than one-third of the distance between the PP and PC veins

863 (ranging from 50 to $130 \mu \mathrm{m}$ ).

Wnt2, Rspo3 and Dkk3 expression in non-parenchymal cells (NPCs)

866 Preparation of probe libraries, hybridization protocol and imaging conditions were 867 previously described ${ }^{19}$. The Aqp1, Igfbp 7 and Ptprb probe libraries were coupled to TMR, 868 the Wnt2 library was coupled to Alexa594 and the Dkk3 or Rspo3 library were coupled to 869 Cy5. Cell membranes were stained with alexa fluor 488 coupled to phalloidin (Rhenium 870 A12379) that was added to GLOX buffer ${ }^{68}$. The central vein was identified based on 871 morphological features inspected in the DAPI and Phalloidin channels and presence of 872 Wnt2-mRNA (detected by smFISH). Central vein niche NPCs were identified by co-

873 staining of Aqp1, Igfbp7 and Ptprb. The central vein area was imaged and the images were 874 analyzed using Image ${ }^{68}$. We counted dots of Wnt2, Rspo3 and Dkk3 expression 875 (corresponding to single mRNA molecules) in NPCs lining the central vein and removed 876 background dots larger than 25 pixels. We then divided the dot count by the segmented 877 cell volume. In total 1420 endothelial cells from 189 central veins of at least 2 mice per 878 time point. In total 1420 NPCs from 189 central veins of at least 2 mice per time point 879 (ZT0,6,12,18) were imaged and a Kruskal-Wallis test based on the mean mRNA dot 880 concentration in each cell was performed to compare the ZT0 and ZT18 time points.

\section{Data availability}

883 scRNA-seq data

884 All scRNA-seq data is deposited in GEO with accession code GSE145197 (reviewer token $885 \mathrm{xxx})$.

886 Reconstructed gene profiles 
887 Reconstructed spatio-temporal gene profiles are available as Matlab files at

888 https://c4science.ch/diffusion/10261/

889 Web-application

890 The whole dataset of gene profiles along with the analysis is available online as a web-

891 application at the URL https://czviz.epfl.ch/. The application was built in Python using the

892 library Dash by Plotly (version 1.0).

\section{Code availability}

894 The code for fitting the mixed-effects models and generating the main figures is available

895 at https://c4science.ch/diffusion/10261/

\section{References}

897 1. Gebhardt, R. Metabolic zonation of the liver: Regulation and implications for liver 898 function. Pharmacol. Ther. 53, 275-354 (1992).

899 2. Hoehme, S. et al. Prediction and validation of cell alignment along microvessels as

900 order principle to restore tissue architecture in liver regeneration. Proc. Natl. Acad. Sci.

901 107, 10371-10376(2010).

902 3. Ben-Moshe, S. \& Itzkovitz, S. Spatial heterogeneity in the mammalian liver. Nat.

903 Rev. Gastroenterol. Hepatol. 16, 395-410 (2019).

904 4. Colnot, S. \& Perret, C. Liver Zonation. in Molecular Pathology of Liver Diseases

905 (ed. Monga, S. P. S.) vol. 5 7-16 (Springer US, 2011).

906 5. Wang, B., Zhao, L., Fish, M., Logan, C. Y. \& Nusse, R. Self-renewing diploid

907 Axin2+ cells fuel homeostatic renewal of the liver. Nature 524, 180-185 (2015).

908 6. Planas-Paz, L. et al. The RSPO-LGR4/5-ZNRF3/RNF43 module controls liver 909 zonation and size. Nat. Cell Biol. 18, 467-479 (2016).

$9107 . \quad$ Jungermann, K. \& Kietzmann, T. Zonation of parenchymal and nonparenchymal

911 metabolism in liver. Annu. Rev. Nutr. 16, 179-203 (1996).

912 8. Halpern, K. B. et al. Single-cell spatial reconstruction reveals global division of

913 labour in the mammalian liver. Nature 542, 352-356 (2017).

$9149 . \quad$ Dibner, C., Schibler, U. \& Albrecht, U. The Mammalian Circadian Timing System:

915 Organization and Coordination of Central and Peripheral Clocks. Annu. Rev. Physiol. 72, $916 \quad 517-549(2010)$. 
917 10. Green, C. B., Takahashi, J. S. \& Bass, J. The Meter of Metabolism. Cell 134, 728$918742(2008)$.

919 11. Yeung, J. \& Naef, F. Rhythms of the Genome: Circadian Dynamics from 920 Chromatin Topology, Tissue-Specific Gene Expression, to Behavior. Trends Genet. TIG $92134,915-926(2018)$.

922 12. Maury, E., Ramsey, K. M. \& Bass, J. Circadian Rhythms and Metabolic Syndrome:

923 From Experimental Genetics to Human Disease. Circ. Res. 106, 447-462 (2010).

924 13. Guan, D. et al. The hepatocyte clock and feeding control chronophysiology of 925 multiple liver cell types. Science 369, 1388-1394 (2020).

926 14. Mermet, J., Yeung, J. \& Naef, F. Systems Chronobiology: Global Analysis of Gene 927 Regulation in a 24-Hour Periodic World. Cold Spring Harb. Perspect. Biol. 9, a028720 928 (2017).

929 15. Zhang, R., Lahens, N. F., Ballance, H. I., Hughes, M. E. \& Hogenesch, J. B. A 930 circadian gene expression atlas in mammals: implications for biology and medicine. Proc.

931 Natl. Acad. Sci. U. S. A. 111, 16219-16224 (2014).

932 16. Sobel, J. A. et al. Transcriptional regulatory logic of the diurnal cycle in the mouse 933 liver. PLOS Biol. 15, e2001069 (2017).

934 17. Vollmers, C. et al. Time of feeding and the intrinsic circadian clock drive rhythms 935 in hepatic gene expression. Proc. Natl. Acad. Sci. U. S. A. 106, 21453-21458 (2009).

936 18. Yeung, J. et al. Transcription factor activity rhythms and tissue-specific chromatin 937 interactions explain circadian gene expression across organs. Genome Res. 28, 182-191 938 (2018).

939 19. Halpern, K. B. et al. Paired-cell sequencing enables spatial gene expression 940 mapping of liver endothelial cells. Nat. Biotechnol. 36, 962-970 (2018).

941 20. Bunger, M. K. et al. Mop3 Is an Essential Component of the Master Circadian 942 Pacemaker in Mammals. Cell 103, 1009-1017 (2000).

943 21. Ripperger, J. A., Shearman, L. P., Reppert, S. M. \& Schibler, U. CLOCK, an 944 essential pacemaker component, controls expression of the circadian transcription factor 945 DBP. Genes Dev. 14, 679-689 (2000).

946 22. Beal, J. Biochemical complexity drives log-normal variation in genetic expression. 947 Eng. Biol. 1, 55-60 (2017). 
948 23. McLean, R. A., Sanders, W. L. \& Stroup, W. W. A Unified Approach to Mixed 949 Linear Models. Am. Stat. 45, 54 (1991).

950 24. Wagenmakers, E.-J. \& Farrell, S. AIC model selection using Akaike weights. 951 Psychon. Bull. Rev. 11, 192-196 (2004).

952 25. Atger, F. et al. Circadian and feeding rhythms differentially affect rhythmic mRNA

953 transcription and translation in mouse liver. Proc. Natl. Acad. Sci. 112, E6579-E6588 954 (2015).

955 26. Kietzmann, T. Metabolic zonation of the liver: The oxygen gradient revisited. 956 Redox Biol. 11, 622-630 (2017).

957 27. Robles, M. S., Cox, J. \& Mann, M. In-Vivo Quantitative Proteomics Reveals a Key 958 Contribution of Post-Transcriptional Mechanisms to the Circadian Regulation of Liver 959 Metabolism. PLoS Genet. 10, e1004047 (2014).

960 28. Mauvoisin, D. et al. Circadian clock-dependent and -independent rhythmic 961 proteomes implement distinct diurnal functions in mouse liver. Proc. Natl. Acad. Sci. 111, 962 167-172 (2014).

963 29. Gebhardt, R. Liver zonation: Novel aspects of its regulation and its impact on 964 homeostasis. World J. Gastroenterol. 20, 8491 (2014).

965 30. Kornmann, B., Schaad, O., Bujard, H., Takahashi, J. S. \& Schibler, U. System966 Driven and Oscillator-Dependent Circadian Transcription in Mice with a Conditionally 967 Active Liver Clock. PLoS Biol. 5, e34 (2007).

968 31. Reinke, H. et al. Differential display of DNA-binding proteins reveals heat-shock

969 factor 1 as a circadian transcription factor. Genes Dev. 22, 331-345 (2008).

970 32. Sinturel, F. et al. Diurnal Oscillations in Liver Mass and Cell Size Accompany 971 Ribosome Assembly Cycles. Cell 169, 651-663.e14 (2017).

972 33. Kietzmann, T., Cornesse, Y., Brechtel, K., Modaressi, S. \& Jungermann, K. 973 Perivenous expression of the mRNA of the three hypoxia-inducible factor alpha-subunits, 974 HIF1alpha, HIF2alpha and HIF3alpha, in rat liver. Biochem. J. 354, 531-537 (2001).

975 34. Ma, D., Panda, S. \& Lin, J. D. Temporal orchestration of circadian autophagy 976 rhythm by $\mathrm{C} / \mathrm{EBP} \beta$ : C/EBP $\beta$ regulates circadian autophagy rhythm. EMBO J. 30, 46429774651 (2011).

978 35. Wang, J. et al. Nuclear Proteomics Uncovers Diurnal Regulatory Landscapes in 
979 Mouse Liver. Cell Metab. 25, 102-117 (2017).

980 36. Harper, J. W., Ordureau, A. \& Heo, J.-M. Building and decoding ubiquitin chains

981 for mitophagy. Nat. Rev. Mol. Cell Biol. 19, 93-108 (2018).

982 37. Le Martelot, G. et al. REV-ERB $\alpha$ Participates in Circadian SREBP Signaling and

983 Bile Acid Homeostasis. PLoS Biol. 7, e1000181 (2009).

984 38. Ferrell, J. M. \& Chiang, J. Y. L. Short-Term Circadian Disruption Impairs Bile

985 Acid and Lipid Homeostasis in Mice. Cell. Mol. Gastroenterol. Hepatol. 1, 664-677

986 (2015).

987 39. Ma, K. et al. Circadian Dysregulation Disrupts Bile Acid Homeostasis. PLoS ONE

988 4, e6843 (2009).

989 40. Yu, L. et al. Disruption of Abcg5 and Abcg8 in mice reveals their crucial role in

990 biliary cholesterol secretion. Proc. Natl. Acad. Sci. 99, 16237-16242 (2002).

991 41. Oki, S. et al. Ch IP -Atlas: a data-mining suite powered by full integration of public

992 Ch IP -seq data. EMBO Rep. 19, (2018).

993 42. Gotic, I. et al. Temperature regulates splicing efficiency of the cold-inducible

994 RNA-binding protein gene Cirbp. Genes Dev. 30, 2005-2017 (2016).

995 43. Jakobsson, A., Jörgensen, J. A. \& Jacobsson, A. Differential regulation of fatty acid 996 elongation enzymes in brown adipocytes implies a unique role for Elovl3 during increased

997 fatty acid oxidation. Am. J. Physiol.-Endocrinol. Metab. 289, E517-E526 (2005).

998 44. Burke, Z. D. \& Tosh, D. The Wnt/ $\beta$-catenin pathway: master regulator of liver 999 zonation? BioEssays 28, 1072-1077 (2006).

1000 45. Gougelet, A. et al. T-cell factor 4 and $\beta$-catenin chromatin occupancies pattern 1001 zonal liver metabolism in mice. Hepatology 59, 2344-2357 (2014).

1002 46. Lavery, D. J. et al. Circadian Expression of the Steroid $15 \alpha$-Hydroxylase (Cyp2a4)

1003 and Coumarin 7-Hydroxylase (Cyp2a5) Genes in Mouse Liver Is Regulated by the PAR

1004 Leucine Zipper Transcription Factor DBP. Mol. Cell. Biol. 19, 6488-6499 (1999).

1005 47. Carmon, K. S., Gong, X., Lin, Q., Thomas, A. \& Liu, Q. R-spondins function as

1006 ligands of the orphan receptors LGR4 and LGR5 to regulate Wnt/beta-catenin signaling.

1007 Proc. Natl. Acad. Sci. U. S. A. 108, 11452-11457 (2011).

1008 48. Aizarani, N. et al. A human liver cell atlas reveals heterogeneity and epithelial 1009 progenitors. Nature 572, 199-204 (2019). 
1010 49. Brosch, M. et al. Epigenomic map of human liver reveals principles of zonated 1011 morphogenic and metabolic control. Nat. Commun. 9, (2018).

1012 50. Mermet, J. et al. Clock-dependent chromatin topology modulates circadian 1013 transcription and behavior. Genes Dev. 32, 347-358 (2018).

1014 51. Le Martelot, G. et al. Genome-Wide RNA Polymerase II Profiles and RNA

1015 Accumulation Reveal Kinetics of Transcription and Associated Epigenetic Changes

1016 During Diurnal Cycles. PLoS Biol. 10, e1001442 (2012).

1017 52. Wang, J. et al. Circadian clock-dependent and -independent posttranscriptional

1018 regulation underlies temporal mRNA accumulation in mouse liver. Proc. Natl. Acad. Sci.

1019 115, E1916-E1925 (2018).

1020 53. Nitzan, M., Karaiskos, N., Friedman, N. \& Rajewsky, N. Gene expression 1021 cartography. Nature (2019) doi:10.1038/s41586-019-1773-3.

1022 54. Ben-Moshe, S. et al. Spatial sorting enables comprehensive characterization of 1023 liver zonation. Nat. Metab. 1, 899-911 (2019).

1024 55. Seglen, P. O. Chapter 4 Preparation of Isolated Rat Liver Cells. in Methods in Cell 1025 Biology vol. 13 29-83 (Elsevier, 1976).

1026 56. Darnell, A. M., Subramaniam, A. R. \& O’Shea, E. K. Translational Control through 1027 Differential Ribosome Pausing during Amino Acid Limitation in Mammalian Cells. Mol. 1028 Cell 71, 229-243.e11 (2018).

1029 57. Clayton, D. F., Harrelson, A. L. \& Darnell, J. E. Dependence of liver-specific 1030 transcription on tissue organization. Mol. Cell. Biol. 5, 2623-2632 (1985).

1031 58. Satija, R., Farrell, J. A., Gennert, D., Schier, A. F. \& Regev, A. Spatial 1032 reconstruction of single-cell gene expression data. Nat. Biotechnol. 33, 495-502 (2015).

1033 59. Loud, A. V. A QUANTITATIVE STEREOLOGICAL DESCRIPTION OF THE 1034 ULTRASTRUCTURE OF NORMAL RAT LIVER PARENCHYMAL CELLS. J. Cell 1035 Biol. 37, 27-46 (1968).

1036 60. Kuhn, N. J., Woodworth-Gutai, M., Gross, K. W. \& Held, W. A. Subfamilies of 1037 the mouse major urinary protein (MUP) multi-gene family: sequence analysis of cDNA 1038 clones and differential regulation in the liver. Nucleic Acids Res. 12, 6073-6090 (1984).

1039 61. Hurst, J. L. et al. Individual recognition in mice mediated by major urinary proteins. 1040 Nature 414, 631-634 (2001). 
1041 62. Ilicic, T. et al. Classification of low quality cells from single-cell RNA-seq data.

1042 Genome Biol. 17, 29 (2016).

1043 63. Maaten, L. van der \& Hinton, G. Visualizing Data using t-SNE. J. Mach. Learn.

1044 Res. 9, 2579-2605 (2008).

1045 64. Müller, S., Scealy, J. L. \& Welsh, A. H. Model Selection in Linear Mixed Models.

1046 Stat. Sci. 28, 135-167 (2013).

1047 65. Schwarz, G. Estimating the Dimension of a Model. Ann. Stat. 6, 461-464 (1978).

1048 66. Jammalamadaka, S. R. \& SenGupta, A. Topics in Circular Statistics. vol. 5

1049 (WORLD SCIENTIFIC, 2001).

1050 67. Chen, E. Y. et al. Enrichr: interactive and collaborative HTML5 gene list 1051 enrichment analysis tool. BMC Bioinformatics 14, 128 (2013).

1052 68. Lyubimova, A. et al. Single-molecule mRNA detection and counting in 1053 mammalian tissue. Nat. Protoc. 8, 1743-1758 (2013).

1054

\section{Acknowledgments}

1056 This work was supported by the Rothschild Caesarea Foundation' fund managed by 1057 Weizmann Institute and EPFL, a Swiss National Science Foundation Grant 1058 310030_173079 (to F.N.), and the EPFL. S.I. is supported by the Henry Chanoch Krenter 1059 Institute for Biomedical Imaging and Genomics, The Leir Charitable Foundations, Richard 1060 Jakubskind Laboratory of Systems Biology, Cymerman-Jakubskind Prize, The Lord Sieff 1061 of Brimpton Memorial Fund, the Wolfson Foundation SCG, the Wolfson Family 1062 Charitable Trust, Edmond de Rothschild Foundations, the I-CORE program of the 1063 Planning and Budgeting Committee and the Israel Science Foundation (grants 1902/12 and 1064 1796/12, the Israel Science Foundation grant no. 1486/16, the Chan Zuckerberg Initiative 1065 grant no. CZF2019-002434, the Broad Institute-Israel Science Foundation grant no. 1066 2615/18, the European Research Council (ERC) under the European Union's Horizon 2020 1067 research and innovation program (grant agreement no. 768956, the Bert L. and N. Kuggie 1068 Vallee Foundation, and the Howard Hughes Medical Institute (HHMI) international 1069 research scholar award. 


\section{Author contributions}

1071 F.N. and S.I. conceived the study. K.B.H., J.E.K. and C.H. prepared all the samples and 1072 performed the experiments. C.D. and F.N designed the modeling. K.B.H., J.E.K., C.H. and 1073 C.D. analyzed the data. M.R. and S.M. assisted with the scRNA-seq and smFISH 1074 experiments. F.N and S.I. supervised the study. C.D., J.E.K. and F.N. wrote the manuscript. 1075 All authors reviewed the manuscript and provided input.

\section{Ethics statement}

1077 All animal care and handling were approved by the Institutional Animal Care and Use 1078 Committee of Weizmann Institute of Science and by the Canton de Vaud laws for animal 1079 protection (authorization VD3197.b).

\section{Competing interests}

1081 The authors declare no competing interests. 